\title{
Multidimensional Analysis of the Role of Charged Multivesicular Body Protein 7 in Pan-Cancer
}

\section{Yu Guo \\ Jian Shi \\ Zeyun Zhao \\ Min Wang}

Department of the General Surgery, Jilin University Second Hospital, Changchun, jilin, People's Republic of China
Correspondence: Jian Shi; Min Wang Department of the General Surgery, Jilin University Second Hospital, No. 218 , Ziqiang Street, Nanguan District, Changchun, Jilin, People's Republic of China

Tel +86 I8I35435372; +86 I5364759940 Email Dr.shi@live.cn; jdeywangmin@I63. com
Background: Charged multivesicular body protein 7 is briefly referred to as CHMP7, and it plays a significant role in the endosomal sorting pathway. CHMP7 can form a complex with ESCRTIII to jointly complete the process of contraction, shear bud neck and final membrane shedding.

Methods: TCGA, GEO and CPTAC were chosen for the analysis of the role of CHMP7 in pan-cancer. Role of CHMP7 in pan-cancer was analyzed using R software and tools such as TIMER, GEPIA, UALCAN, String and DiseaseMeth. It includes differential expression analysis of CHMP7, survival analysis, genetic variation analysis, DNA methylation analysis, post-translationally modified protein phosphorylation analysis and functional enrichment analysis.

Results: CHMP7 presents low expression in the majority of tumor tissues and the prognosis is poor in the low expression group. The common gene mutation in CHMP7 is deep deletion, which may lead to frameshift mutations, resulting in a poor prognosis. Functional alterations due to DNA methylation and post-transcriptional protein modifications may be closely associated with tumors. GO analysis revealed that CHMP7-related genes are involved in the composition of the various ESCRT complexes. In terms of molecular function, they mainly bind to GTP, exert GTPase activity and promote multivesicular bodies assembly. In the KEGG enrichment analysis, the main pathways expressed by CHMP7 and related genes were endocytosis, gap junction and phagosome.

Conclusion: Pan-cancer analysis showed that CHMP7 expression was statistically correlated with clinical prognosis, DNA methylation, protein phosphorylation and immune cell infiltration, which may provide new ideas or targets for the diagnosis or treatment.

Keywords: pan-cancer, CHMP7, endosomal sorting complex required for transport, ESCRT, prognosis

\section{Introduction}

Charged multivesicular body protein 7 is briefly referred to as CHMP7, and it plays a significant role in the endosomal sorting pathway. ${ }^{1-3}$ The endosomal sorting complex required for transport (ESCRT) system is the molecular machinery that completes the invagination of endosomal membranes to form multivesicular bodies (MVBs) in eukaryotic cells, and its main function is to promote the degradation of membrane proteins tagged with biquitin. ${ }^{4-6}$ CHMP7 can form a complex with ESCRTIII to jointly complete the process of contraction, shear bud neck and final membrane shedding. ${ }^{2}$

The ESCRT system is an important molecular machinery for membrane protein sorting in eukaryotic cells. ${ }^{7,8}$ It has been shown that ubiquitin-tagged membrane 
proteins are first transported to the endosomal membrane by cytokinesis and then invaginated by the ESCRT system, which releases membrane components containing these proteins into the endosomal lumen to form intralumenal vesicles (ILVs). The endosomes at this point are called MVBs. Later, through fusion with lysosomes, the endosomal vesicles and their membrane-bound proteins are degraded. ${ }^{9,10}$ Epidermal growth factor receptor (EGFR), for example, is degraded through this pathway. In addition to the formation of MVBs, ESCRT is also involved in cellular life activities such as cytoplasmic segregation, budding of vesicular viruses such as HIV, ${ }^{11}$ and cellular autophagy. ${ }^{12}$ Thus, aberrant expression of CHMP7 in various tissues may lead to dysfunction of the ESCRT system, resulting in impaired protein degradation and consequent disease. Malik et $\mathrm{al}^{13}$ performed a transcriptome analysis of primary embryonic motor neurons from mice with spinal and bulbar muscular atrophy (SBMA) and concluded that CHMP7 may play a causal role in disease pathogenesis by disrupting the endosomal-lysosomal system. In addition, Huntington's disease and Parkinson's disease are characterized by the accumulation of intracellular ubiquitinated protein aggregates, a characteristic lesion that may be associated with the accumulation of MVB due to loss of ESCRT function. ${ }^{14,15}$

Regarding tumor research, due to the key role of ESCRT in cell surface receptor degradation, various studies have been conducted to demonstrate the oncogenic or tumor suppressor function of ESCRT members. Among those whose expression was upregulated were Hrs in colorectal adenocarcinoma (COAD) and melanoma; ${ }^{16}$ TSG101 in breast cancer $^{17}$ and papillary thyroid cancer; ${ }^{18}$ and CHMP4C in ovarian cancer. ${ }^{19}$ In contrast, Vps37A was decreased in hepatocellular carcinoma; ${ }^{20}$ UBAP1 expression levels were decreased in nasopharyngeal carcinoma and CHMP1A in pancreatic ductal adenocarcinoma. $^{21}$

The tumor microenvironment (TME) is a complex integrated system formed by the interaction of tumor cells with surrounding tissues and immune cells. ${ }^{22}$ The existence of TME can enhance tumor cell proliferation, migration and immune escape ability, thereby promoting the occurrence and development of tumors. ${ }^{23}$ As a part of TME, infiltrating immune cells have been paid more and more attention by researchers. The immune infiltration of TME provides us with clues to understand the progress of cancer diseases, which is of great significance for tumor prognosis or prediction, and can even become a means of cell therapy. ${ }^{24}$

As an instrumental member involved in the completion of ESCRT, CHMP7 has been the subject of very few studies in oncology. The rapid development of bioinformatics and microarray technologies are widely used for mutation screening at the genomic level, which allows us to use large data in online databases to investigate whether the role of CHMP7 in tumorigenesis development is angelic or demonic. In the article, $\mathrm{R}$ software and databases such as UALCAN, PrognoScan and GEPIA were used to analyze the data from TCGA to visualize the differences in CHMP7 expression in tumor tissues and normal tissues. Then, TIMER and GEPIA databases were used to explore the potential relationship between CHMP7 expression and immune infiltration levels. R software was used to further analyze the correlation of CHMP7 with immune checkpoint molecules and mismatch repair genes in various tumor tissues. It is shown that CHMP7 is lowly expressed in a variety of tumors, and may contribute to poor prognosis of cancer patients through interaction with infiltrating immune cells.

\section{Methods}

\section{Data Sources}

The Cancer Genome Atlas (TCGA, https://www.cancer. gov/about-nci/organization/ccg/research/structuralgenomics/tcga) $)^{25}$ is a cancer research project jointly established by National Cancer Institute (NCI) and National Human Genome Research Institute (NHGRI). Gene Expression Omnibus (GEO) ${ }^{26}$ is a gene expression database created and maintained by the National Center for Biotechnology Information (NCBI, https://www.ncbi.nlm. nih.gov/geo/). It was founded in 2000 and contains highthroughput gene expression data submitted by research institutions around the world.

\section{Analysis of CHMP7 Expression Differences}

The Tumor Immune Estimation Resource (TIMER, https:// cistrome.shinyapps.io/timer/ $^{27}$ database uses highthroughput sequencing (RNA-Seq expression profile) data to analyze the infiltration of immune cells in tumor tissues. The TIMER database was used to analyze the expression levels of CHMP7 in tumor tissues and to compare them with the corresponding normal tissues. 
The Gene Expression Profilling Interactive Analysis (GEPIA, http://gepia.cancer-pku.cn/) database integrates current cancer genomics data, making it easier and faster to mine data and perform dynamic analysis of gene expression profile data. The GEPIA database was selected to analyze the differential expression of CHMP7 in bladder urothelial carcinoma (BLCA), esophageal carcinoma (ESCA), prostate adenocarcinoma (PRAD), thyroid carcinoma (THCA), uterine corpus endometrial carcinoma (UCEC), lymphoid neoplasm diffuse large B-cell lymphoma (DLBC), acute myeloid leukemia (LAML) and lung adenocarcinoma (LUAD). At the protein level, the UALCAN database was employed to visualize the differences in CHMP7 expression between normal and tumor tissues. The Human Protein Atlas provides immunohistochemical evidence of differential expression of CHMP7 in normal and tumor tissues at the pathological level. ${ }^{28-30}$ $\mathrm{R}$ software (version 3.6.3) was employed to further explore the correlation between CHMP7 expression levels and clinicopathological features.

\section{Survival Analysis}

The SURVIVAL package in the R software (version 3.6.3) was used to analyze the correlation between CHMP7 expression and survival in pan-cancer with a risk table. In addition, the Prognoscan database contains a subset of available datasets from the GEO database and related prognostic information. CHMP7 expression levels were looked up in the available microarray datasets of Prognoscan to determine its relationship with prognosis. Cox P-value $<0.05$ was considered significant. We analyzed the correlation between CHMP7 expression and prognosis in breast invasive carcinoma (BRCA), ESCA, head and neck squamous cell carcinoma (HNSC), kidney clear cell carcinoma (KIRC), LAML, sarcoma (SARC), glioblastoma multiforme (GBM) and low-grade glioma of the brain (LGG), and skin cutaneous melanoma (SKCM), calculating hazard ratios (HR) with $95 \%$ confidence intervals $(\mathrm{CI})$ and log-rank $\mathrm{P}$ values.

\section{Mutation Analysis}

The cBioPortal for Cancer Genomics (https://www.cbiopor tal.org $/)^{31}$ provides a web resource for exploring, visualizing and analyzing multidimensional cancer genomic data. The portal reduces cancer tissues and cell lines into molecular expression profiling data to understandable genetic, epigenetic, gene expression and protein events. The interactive query interface integrates user data and allows researchers to interactively explore genetic alterations in different samples, genes, and pathways.

The cBioPortal website was queried for the mutation characteristics of CHMP7 in different cancers and to observe the mutation types and alteration frequencies in all TCGA tumors. The "Comparison" module provides data on whether CHMP7 mutations have an impact on the prognosis of patients with TCGA cancers.

\section{Methylation Analysis}

The correlation between CHMP7 methylation and tumors was analyzed using R software (version 3.6.3) and visualized by choosing the ggplot2 package (version 3.3.3).

The DiseaseMeth (/bio-bigdata.hrbmu.edu.cn/disease meth/) is an abnormal methylation database focusing on human diseases. By integrating public disease methylation big data including TCGA, IHEC, etc., the abnormally methylated genes in diseases are re-identified, and new abnormally methylated genes reported in the literature in recent years are manually searched, including a total of 32,701 sample numbers of methylation profiles, 88 diseases, and 679,602 pairs of methylation-related disease gene relationships. The website was used to explore the correlation between CHMP7 methylation and cancer.

\section{Phosphorylation Analysis}

The UALCAN (http://ualcan.path.uab.edu/index.html) is an effective website for online analysis and mining of cancer data, mainly based on relevant cancer data from the TCGA database. The CPTAC dataset was picked to compare the differences in CHMP7 phosphorylation levels in normal and tumor tissues.

\section{Immuno-Infiltration Analysis}

$\mathrm{R}$ software (version 3.6.3) was employed to analyze the correlation of CHMP7 with immune checkpoint molecules and mismatch repair genes in various tumor tissues, and the ggplot2 package (version 3.3.3) was chosen for visualization.

\section{Enrichment Analysis}

The GEPIA and the String were employed to screen for genes associated with CHMP7 expression or function. The resulting gene sets were subjected to functional clustering analysis. Gene Ontology analysis (GO) is an internationally standardized gene function classification system that provides a set of dynamically updated controlled vocabulary to comprehensively describe the attributes of genes 
and gene products in organisms. ${ }^{32}$ KEGG is a database based on human pathways.

\section{Statistical Analysis}

The Log rank test was applied to compare survival differences between high and low expression groups, univariate Cox analysis was performed to determine the prognostic impact of low CHMP7 expression on COAD patients, and Spearman correlation was used to evaluate the correlation of gene expression. The statistical analyses involved in this article, such as differential expression of CHMP7 in normal and tumor tissues, survival analysis of CHMP7 high and low expression groups, and the correlation of CHMP7 with immune checkpoint and mismatch repair genes with $\mathrm{p}$-values $<0.05$ were considered significant.

\section{Results}

\section{Gene Expression}

The expression of CHMP7 in different TCGA tumor databases was analyzed in TIMER2, as shown in Figure 1A. The results demonstrated that in BLCA, COAD, KICH, KIRC, KIRP, LUSC, PRAD, THCA and UCEC, the CHMP7 expression levels in tumor tissues were significantly lower than those in normal tissues $(p<0.05)$. In contrast, in CHOL, HNSC and LIHC, the CHMP7 expression levels in tumor tissues were markedly higher than those in normal tissues $(\mathrm{p}<0.05)$.

Considering the small amount of information on normal samples included in the TCGA database, the data of GTEx were counted together. The GEPIA was employed for the analysis and CHMP7 was significantly low expressed in tumor tissues in BLCA, ESCA, PRAD, THCA and UCEC, while the opposite results were observed in LUAD, LAML and DLBC $(\mathrm{P}<0.05$; Figure 1B-I).

At the protein expression level, the CPTAC dataset revealed that CHMP7 was lowly expressed in tumor tissues in BRAC ( $\mathrm{P}=3.38 \mathrm{e}-05), \operatorname{COAD}(\mathrm{P}=1.49 \mathrm{e}-05)$ and UCEC ( $\mathrm{P}=3.61 \mathrm{e}-03)$, while it was highly expressed in LUAD ( $\mathrm{P}=4.57 \mathrm{e}-03)$, which was consistent with the results of the above gene expression analysis (Figure 1J-M).

The HPA database is based on proteomic, transcriptomic and systems biology data and includes protein expression in tumor and normal tissues. CHMP7 is generally medium expressed in normal tissues such as stomach, kidney, testis and lung, while it is highly expressed in colon tissues. In STAD, TGCT, COAD, LUAD and
KIRC, CHMP7 is lowly expressed (Figure 2A-E). The low expression level of CHMP7 is closely associated with the staging of many tumors. In BLCA, COAD, KIRC, SKCM, THCA and UCS, patients with lower CHMP7 levels showed more advanced tumor staging ( $\mathrm{P}$ $<0.05$; Figure 3A-F).

\section{Survival Analysis}

The tumor data in TCGA were divided into low and high expression groups according to CHMP7 expression level to explore the correlation between CHMP7 expression level and prognosis, and OS served as an observational index. In BRCA ( $\mathrm{P}=0.018)$, ESCA $(\mathrm{P}=0.037)$, HNSC ( $\mathrm{P}$ $=0.031), \operatorname{KIRC}(P=0.004)$, SARC $(P=0.03)$, GBM + LGG $(\mathrm{P}=0.003)$ and SKCM $(\mathrm{P}=0.03)$, CHMP7 low expression group had poorer prognosis and shorter OS. In reverse, in LAML $(\mathrm{P}=0.029)$, the prognosis was better in the CHMP7 low-expression group (Figure 4A-H).

The Prognoscan website was used to explore the correlation between tumor prognosis and CHMP7 in the GEO database. Some of the results are shown in Figure 2. In BLCA (GSE5287; cox P = 0.008926), BRCA (GSE3143; $\operatorname{cox} \mathrm{P}=1.3 \mathrm{e}-03$ and GSE7390; $\operatorname{cox} \mathrm{P}=0.01934)$, COAD (GSE12945; cox P = 0.035903) and LUAD (jacob-00182CANDF; cox $\mathrm{P}=0.026792$ ), low CHMP7 expression was significantly associated with poor prognosis of patients (Figure 4I-N).

\section{Mutation Analysis}

We analyzed the CHMP7 genetic mutations in the TCGA dataset. The results showed that the frequency of CHMP7 mutation was higher in PRAD, OV, and LIHC, 7\% and $8 \%$, respectively. The most common mutation was deep deletion, which is shown in blue in Figure 5A. Mutation also occurred more frequently in UCEC. In PCPG, the main type of mutation in CHMP7 was amplification. In Figure 5B, the common mutation types and sites of CHMP7 are shown. Missense mutations were found to be the main type of CHMP7 mutations, mainly occurring in the Snf7 region. The three-dimensional structure of the protein is also shown schematically in the Figure 5C. To further explore the correlation between CHMP7 gene mutations and prognosis of patients with different tumors. The results demonstrated that in patients with COAD and UCEC, the CHMP7 gene mutation group resulted in poor DFS ( $\log \mathrm{P}=0.0170 ; \log \mathrm{P}=4.41 \mathrm{e}-08)$ without significant effect on $\mathrm{OS}(\log \mathrm{P}=0.176 ; \log \mathrm{P}=0.982)$. In the 


\section{GENE EXPRESSION}
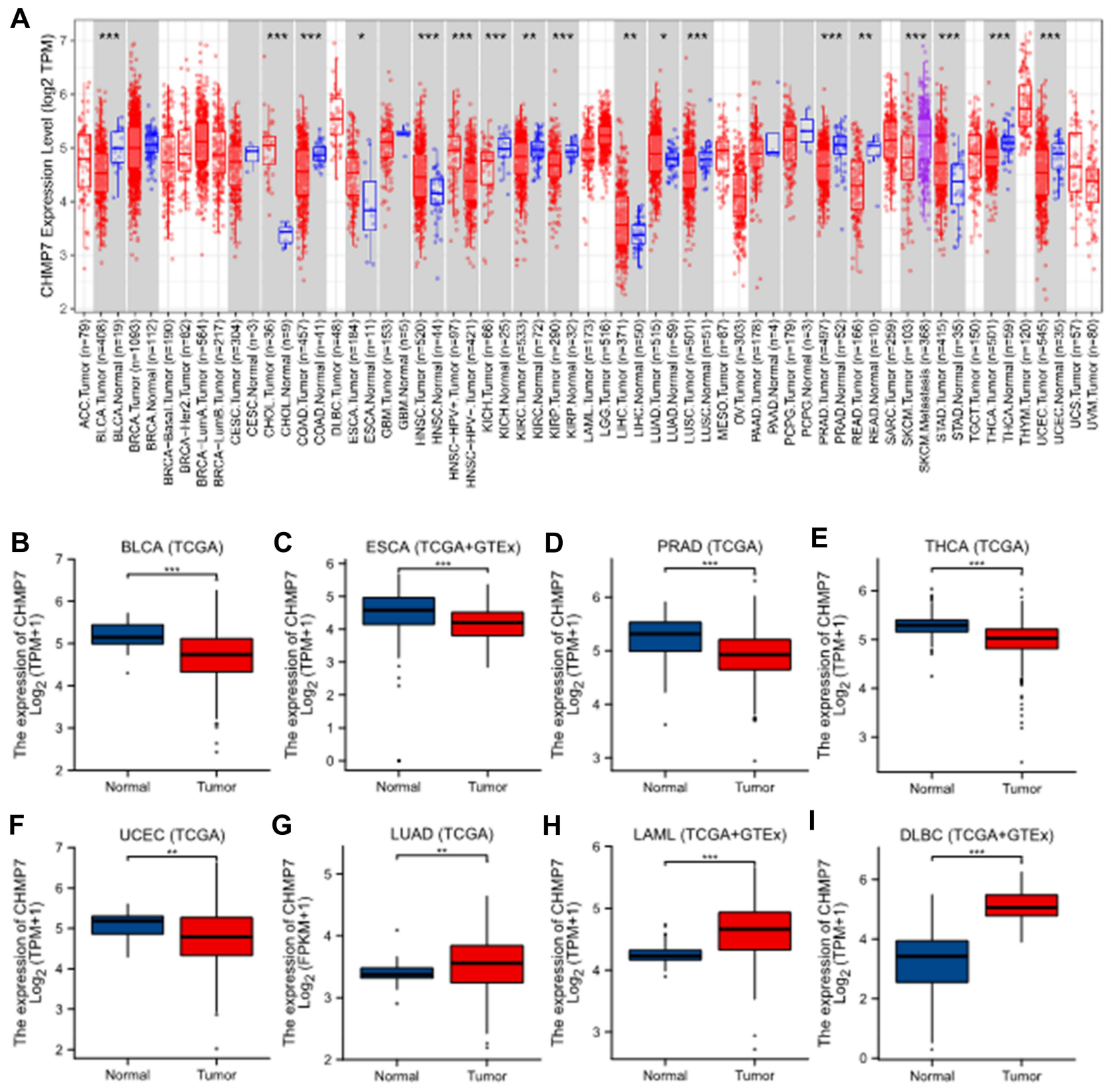

\section{PROTEIN EXPRESSION}
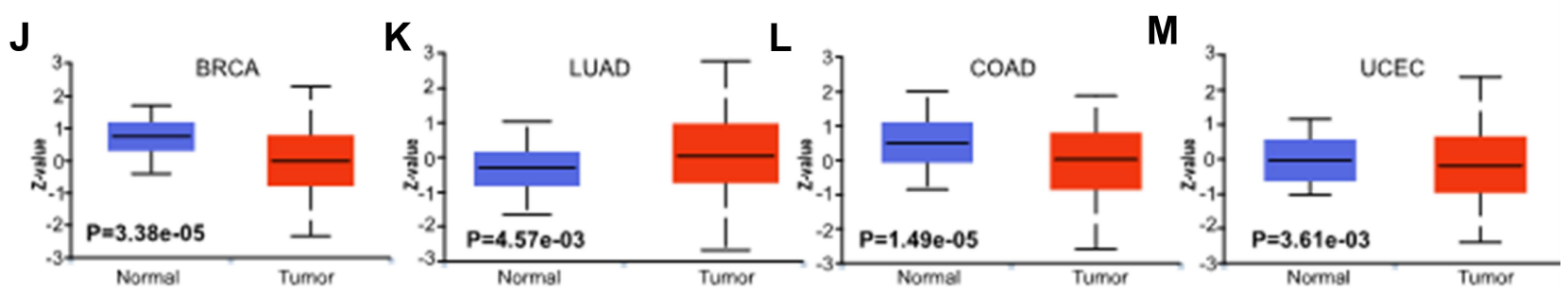

Figure I CHMP7 expression level. (A) the differential expression of CHMP7 in tumor tissues and adjacent tissues; (B-I) Differential expression of CHMP7 in BLCA, ESCA, PRAD, THCA, UCEC, LUAD, LAML and DLBC in the TCGA merged GTEx dataset; (J-M) Differential expression of CHMP7 protein in BRCA, LUAD, KIRC and UCEC and corresponding normal tissues in the CPTAC dataset(*: $\mathrm{P}<0.05$; **: $\mathrm{P}<0.0 \mathrm{I}$; ***: $\mathrm{P}<0.00 \mathrm{I})$. 
A

\begin{tabular}{|l|}
\hline Stomach tissue \\
\hline HPA036119 \\
Male, age 72 \\
Normal tissue \\
Patient id : 2583 \\
\\
\hline Glandular cells \\
Staining: Medium \\
Intensity: Moderate \\
\hline
\end{tabular}

B

\begin{tabular}{|l|}
\hline Kidney tissue \\
\hline HPA036119 \\
Female, age 41 \\
Normal tissue \\
Patient id : 2530 \\
\hline Glomeruli cells \\
Staining: Medium \\
Intensity: Moderate \\
\hline
\end{tabular}

C

\begin{tabular}{|c|}
\hline Testis tissue \\
\hline HPA036119 \\
Male, age 46 \\
Normal tissue \\
Patient id : 2435 \\
\\
\hline Leydig cells \\
Staining: Medium \\
Intensity: Moderate \\
\hline
\end{tabular}

D

\begin{tabular}{|c|}
\hline Colon tissue \\
\hline HPA036119 \\
Female, age 82 \\
Normal tissue \\
Patient id : 2960 \\
\\
Endothelial cells \\
\hline Staining: High \\
Intensity: Strong \\
\hline
\end{tabular}

E

\begin{tabular}{|c|}
\hline Lung tissue \\
\hline HPA036119 \\
Female, age 49 \\
Normal tissue \\
Patient id : 2268 \\
\\
\hline Alveolar cells \\
Staining: Medium \\
Intensity: Moderate \\
\hline
\end{tabular}
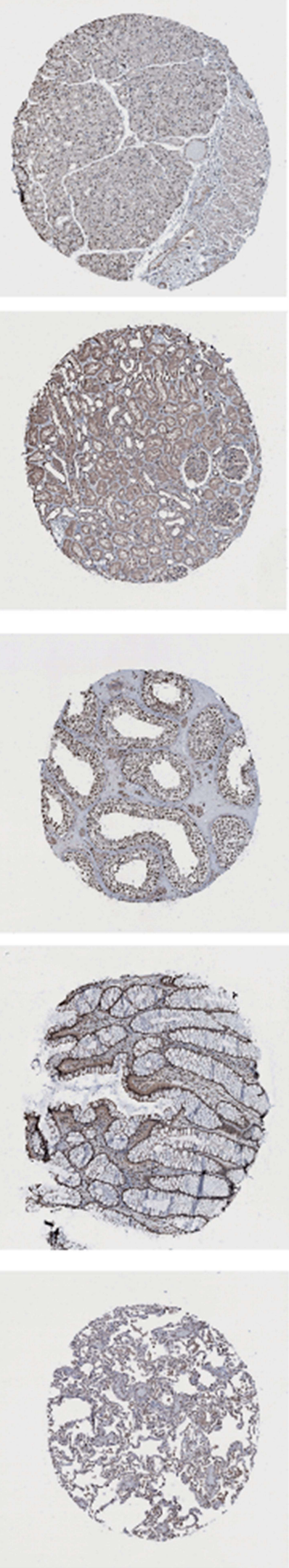
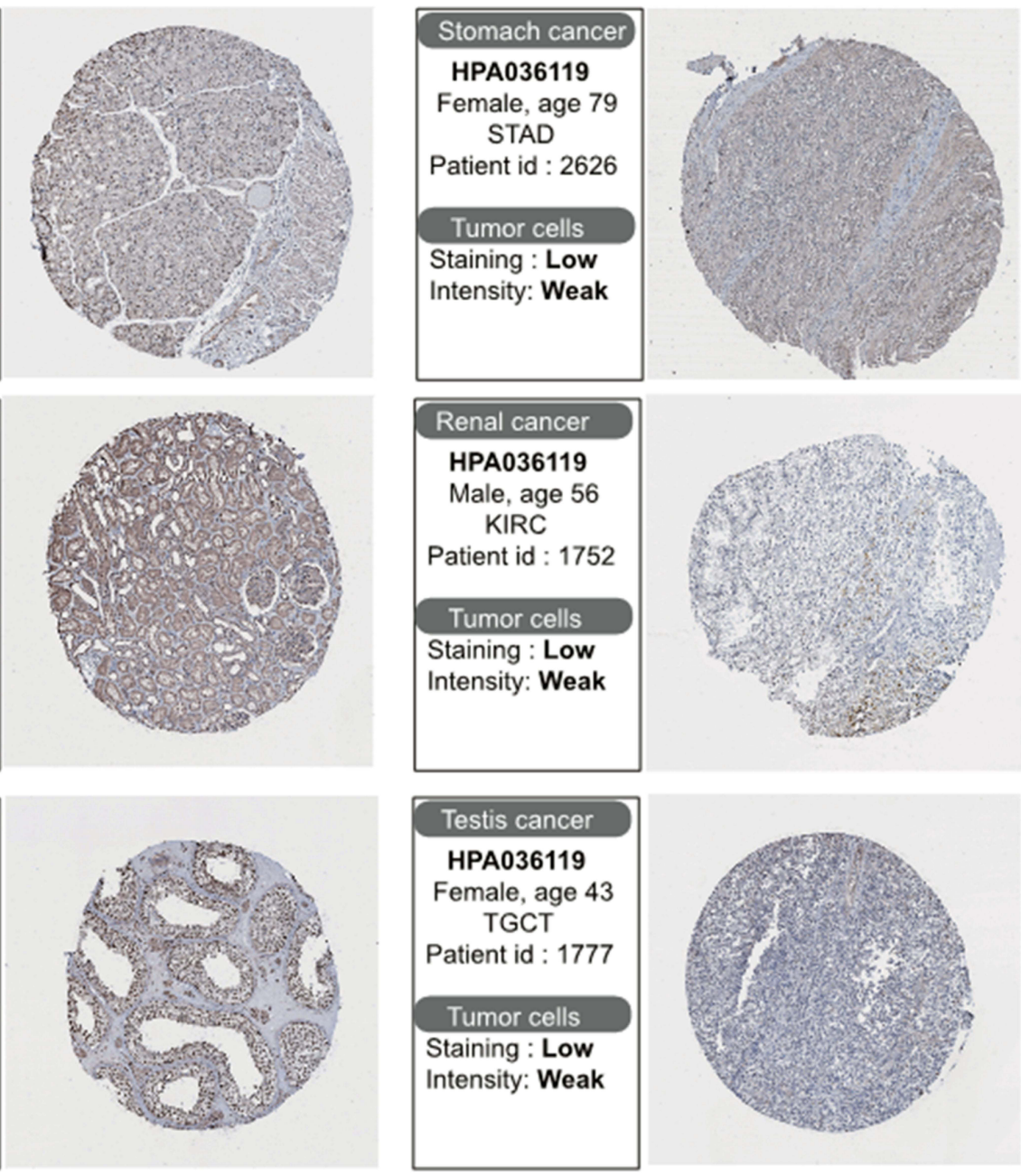

\begin{tabular}{|l|}
\hline Renal cancer \\
\hline HPA036119 \\
Male, age 56 \\
KIRC \\
Patient id : 1752 \\
\\
Tumor cells \\
Staining : Low \\
Intensity: Weak \\
\hline
\end{tabular}
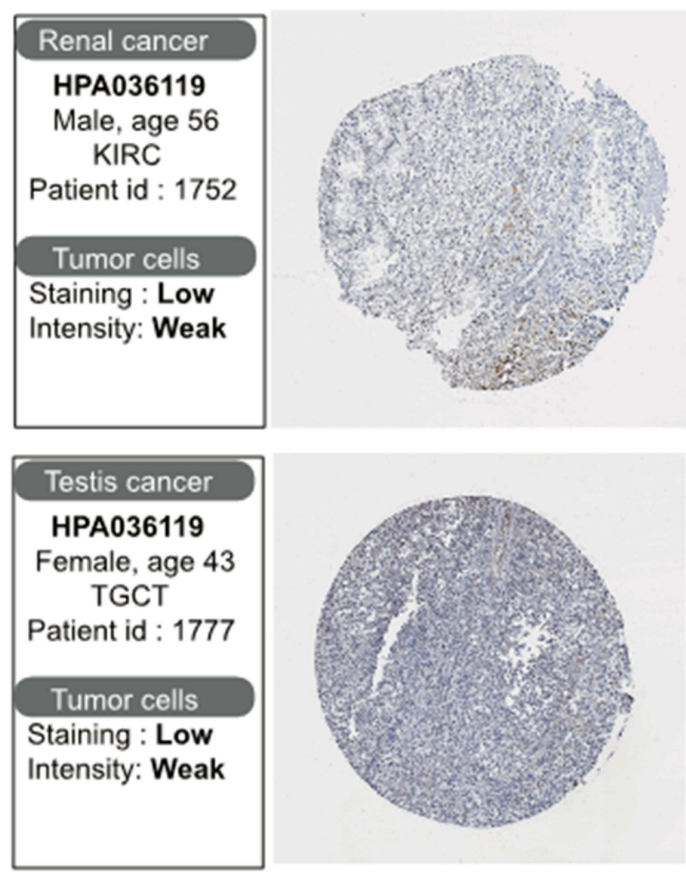

\begin{tabular}{|l|}
\hline Colon cancer \\
\hline HPA036119 \\
Male, age 71 \\
COAD \\
Patient id : 2626 \\
\\
Tumor cells \\
Staining : Low \\
Intensity: Moderate \\
\hline
\end{tabular}

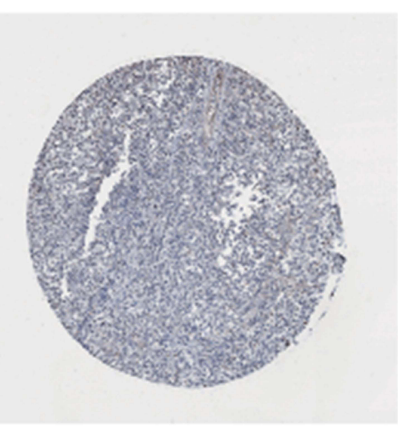

\begin{tabular}{|l|}
\hline Lung cancer \\
\hline HPA036119 \\
Female, age 63 \\
LUAD \\
Patient id : 1765 \\
\\
\hline Tumor cells \\
Staining : Low \\
Intensity: Moderate \\
\hline
\end{tabular}

Figure 2 Differential expression of protein levels of CHMP7 in the HPA database. (A) CHMP7 was moderately expressed in normal gastric tissues and lowly expressed in STAD tissues; (B) CHMP7 was moderately expressed in normal kidney tissues and lowly expressed in KIRC tissues; (C) CHMP7 was moderately expressed in normal testicular tissues and lowly expressed in TGCT tissues; (D) CHMP7 was highly expressed in normal colon tissues and lowly expressed in COAD tissues; (E) CHMP7 was moderately expressed in normal lung tissues and lowly expressed in LUAD tissues. 
A

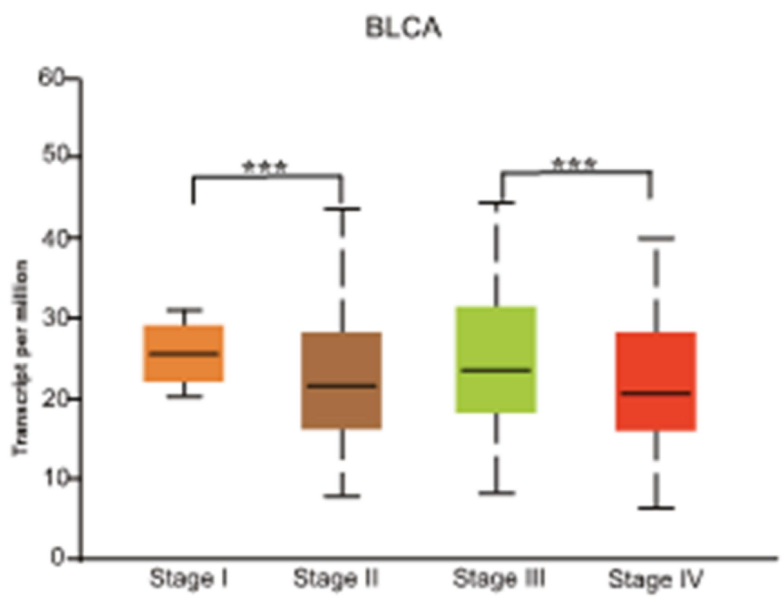

C

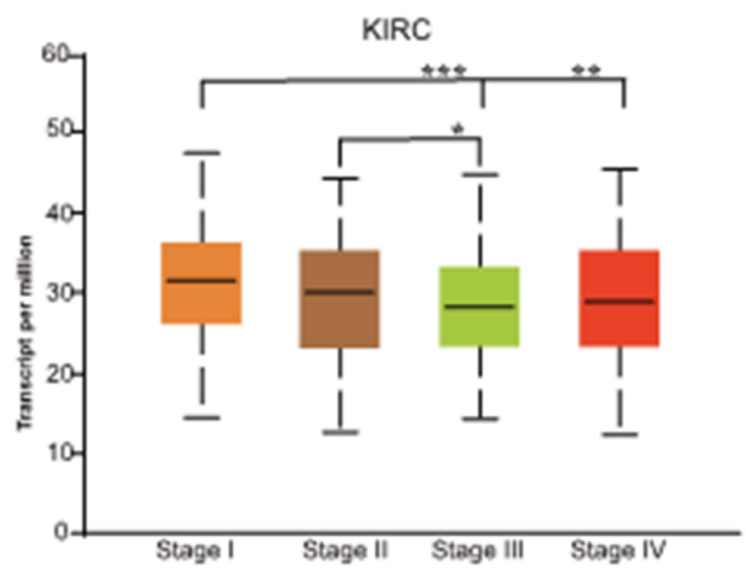

E

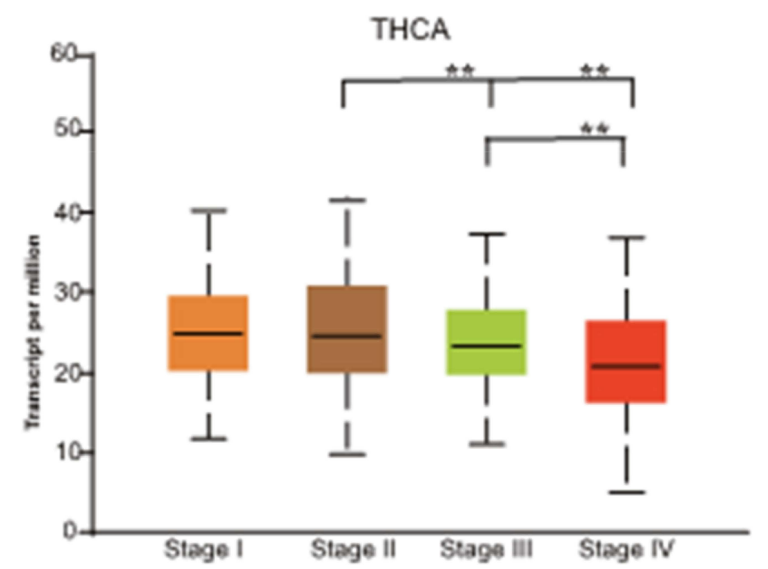

B

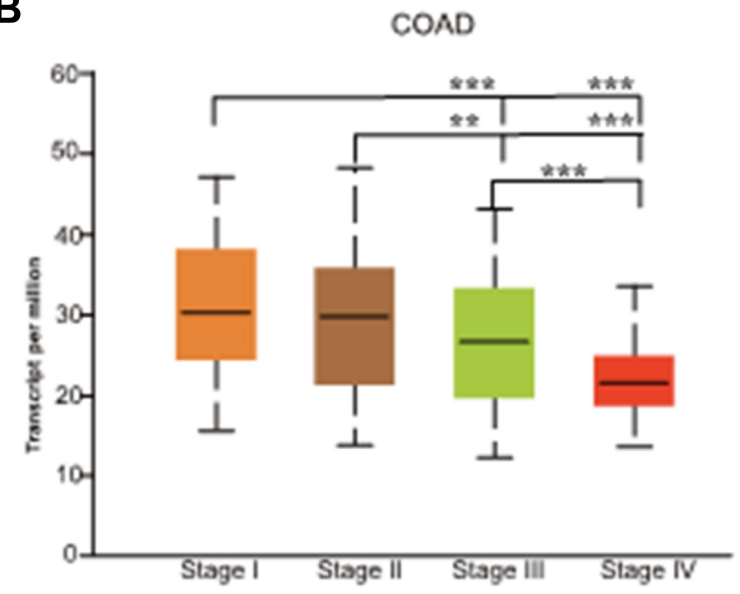

D

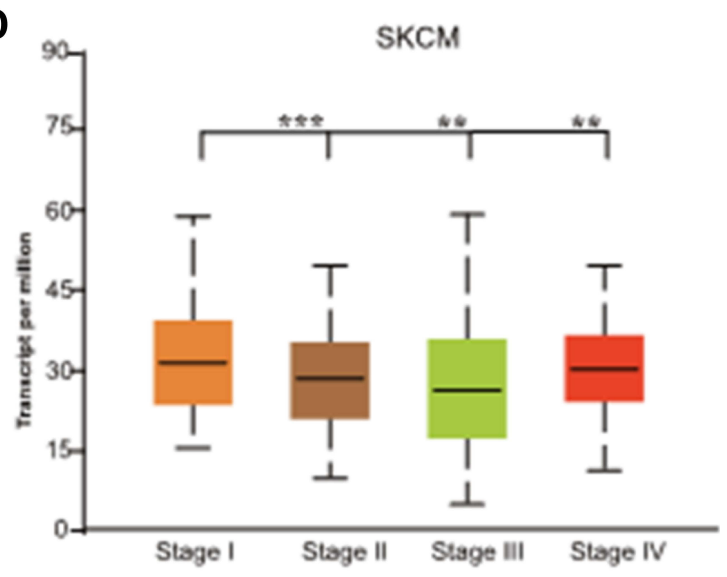

$\mathbf{F}$

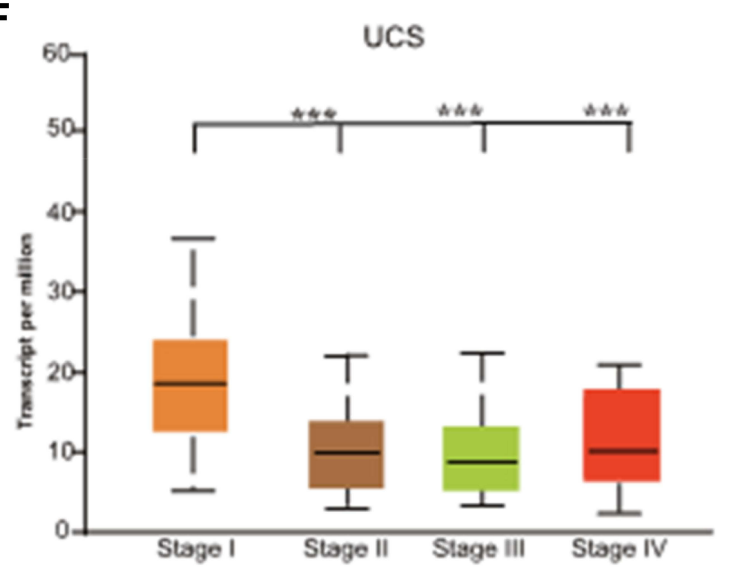

Figure 3 Correlation analysis of CHMP7 expression levels with clinicopathological staging. (A-F): In BLCA, COAD, KIRC, SKCM, THCA and UCS, patients with lower CHMP7 levels showed more advanced tumor staging (*P $<0.05 ; * * \mathrm{P}<0.0 \mathrm{I}$; ***P $<0.00 \mathrm{I})$.

remaining tumor patients, CHMP7 gene mutation had no significant effect on prognosis ( $P>0.05$; Figure $5 \mathrm{D}$ and $\mathrm{E})$.

\section{Methylation Analysis}

$\mathrm{R}$ software (version 3.6.3) was deployed to investigate the potential correlations between CHMP7 DNA methylation and different tumor pathogenesis. Significant negative correlation was observed between CHMP7 DNA methylation and probe cg00140501 in multiple tumor tissues of TCGA. As shown in Figure 6A-J, CHMP7 DNA methylation was significantly negatively correlated with probe $\operatorname{cg} 00140501$ in ACC $(\mathrm{R}=-0.250 ; \mathrm{P}=0.028)$, $\mathrm{BLCA}(\mathrm{R}=-0.140 ; \mathrm{P}=$ 

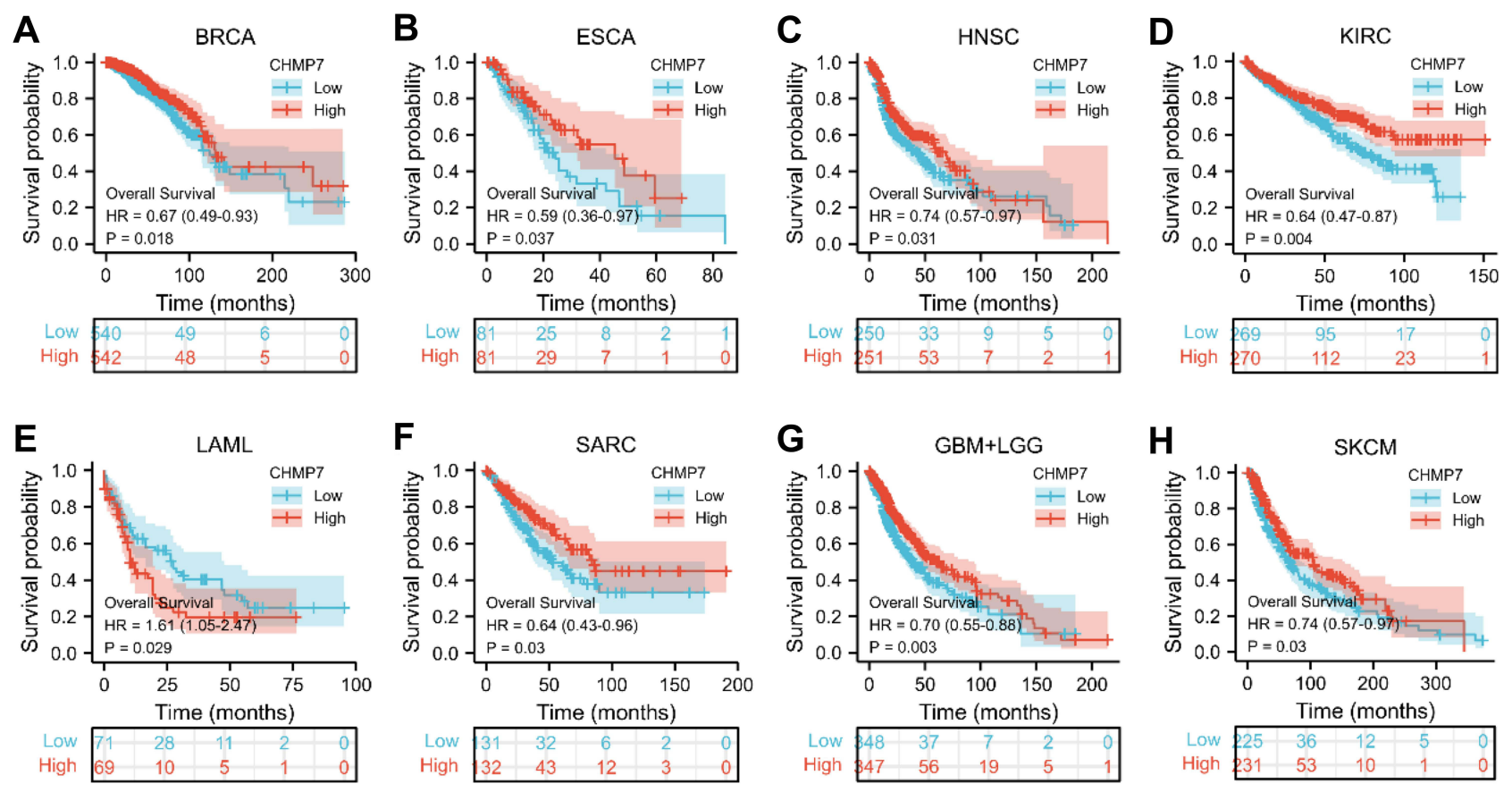

I

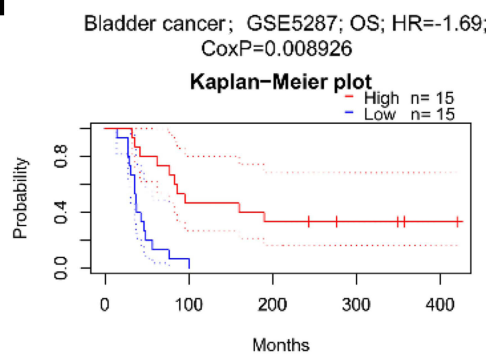
L Breast cancer; GSE7390; OS; HR=--1.01;

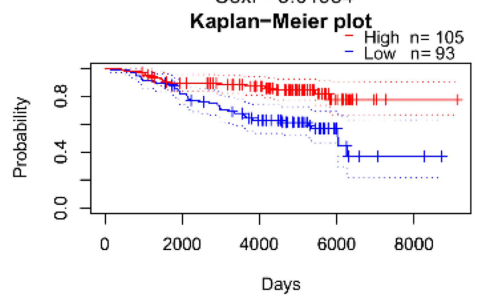

J

J Brain cancer; GSE4412; OS; HR=-1.48; CoxP $=0.002089$

Kaplan-Meier plot

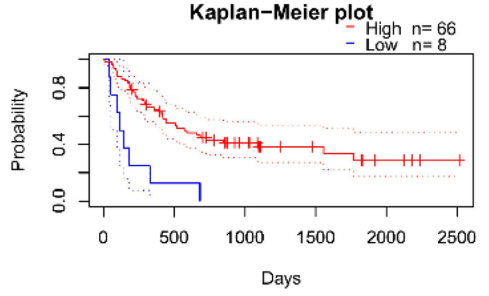

M Colorectal cancer; GSE12945; OS; HR=-1.01; CoxP $=0.035903$

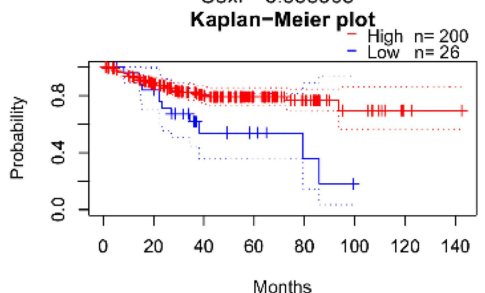

K Breast cancer; GSE3143; OS; HR=-1.16 $\operatorname{CoxP}=0.000137$ Kaplan-Meier plot

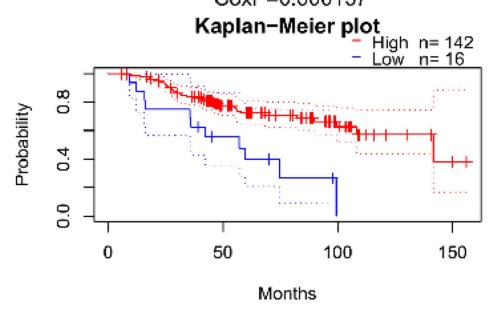

N Lung cancer; jacob-00182-CANDF; OS; $H R=-1.58$; $\operatorname{CoxP}=0.026792$ Kaplan-Meier plot

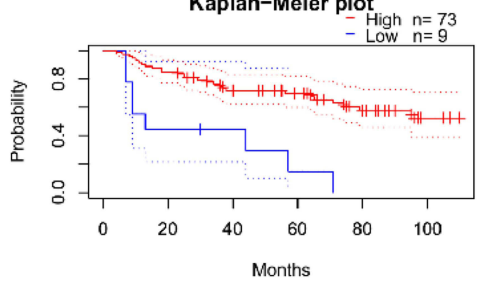

Figure 4 Survival analysis. $(A-H)$ In BRCA $(P=0.018)$, ESCA $(P=0.037)$, HNSC $(P=0.031)$, KIRC $(P=0.004)$, SARC $(P=0.03)$, GBM+LGG $(P=0.003)$ and SKCM $(P=$ $0.03)$, CHMP7 low expression group had poorer prognosis and shorter $O S$. In reverse, in $L A M L(P=0.029)$, the prognosis was better in the CHMP7 low expression group; I-N: In BLCA (GSE5287; cox P = 0.008926), BRCA (GSE3।43; cox P = 1.3e-03 and GSE7390; cox P = 0.01934), COAD (GSEI2945; cox P = 0.035903) and LUAD (jacob00 I82-CANDF; cox $\mathrm{P}=0.026792$ ), low CHMP7 expression was significantly associated with poor prognosis of patients.

0.003), BRCA $(\mathrm{R}=-0.170 ; \mathrm{P}<0.001), \operatorname{COAD}(\mathrm{R}=$ $-0.200 ; \mathrm{P}=0.001)$, LIHC $(\mathrm{R}=-0.190 ; \mathrm{P}<0.001)$, LUAD $(\mathrm{R}=-0.093 ; \mathrm{P}=0.048)$, LUSC $(\mathrm{R}=-0.180$; $\mathrm{P}<0.001)$, PRAD $(\mathrm{R}=-0.180 ; \mathrm{P}<0.001)$, SKCM $(\mathrm{R}=$ $-0.280 ; \mathrm{P}<0.001)$ and STAD $(\mathrm{R}=-0.230 ; \mathrm{P}<0.001)$.

The DiseaseMeth database was used to explore the relationship between CHMP7 DNA methylation and different tumor tissues. In $\operatorname{COAD}(\mathrm{P}=1.14 \mathrm{e}-06)$, LGG ( $\mathrm{P}=3.79 \mathrm{e}-09), \mathrm{KICH}(\mathrm{P}=4.22 \mathrm{e}-05), \mathrm{ACC}(\mathrm{P}$ $=2.11 \mathrm{e}-06)$ and UCS $(\mathrm{P}=1.60 \mathrm{e}-03)$, CHMP7 DNA methylation was significantly negatively correlated with probe NM_001317899; in TGCT ( $\mathrm{P}=1.32 \mathrm{e}-15)$, it was negatively correlated with probe NM_152272 (Figure 6K). 

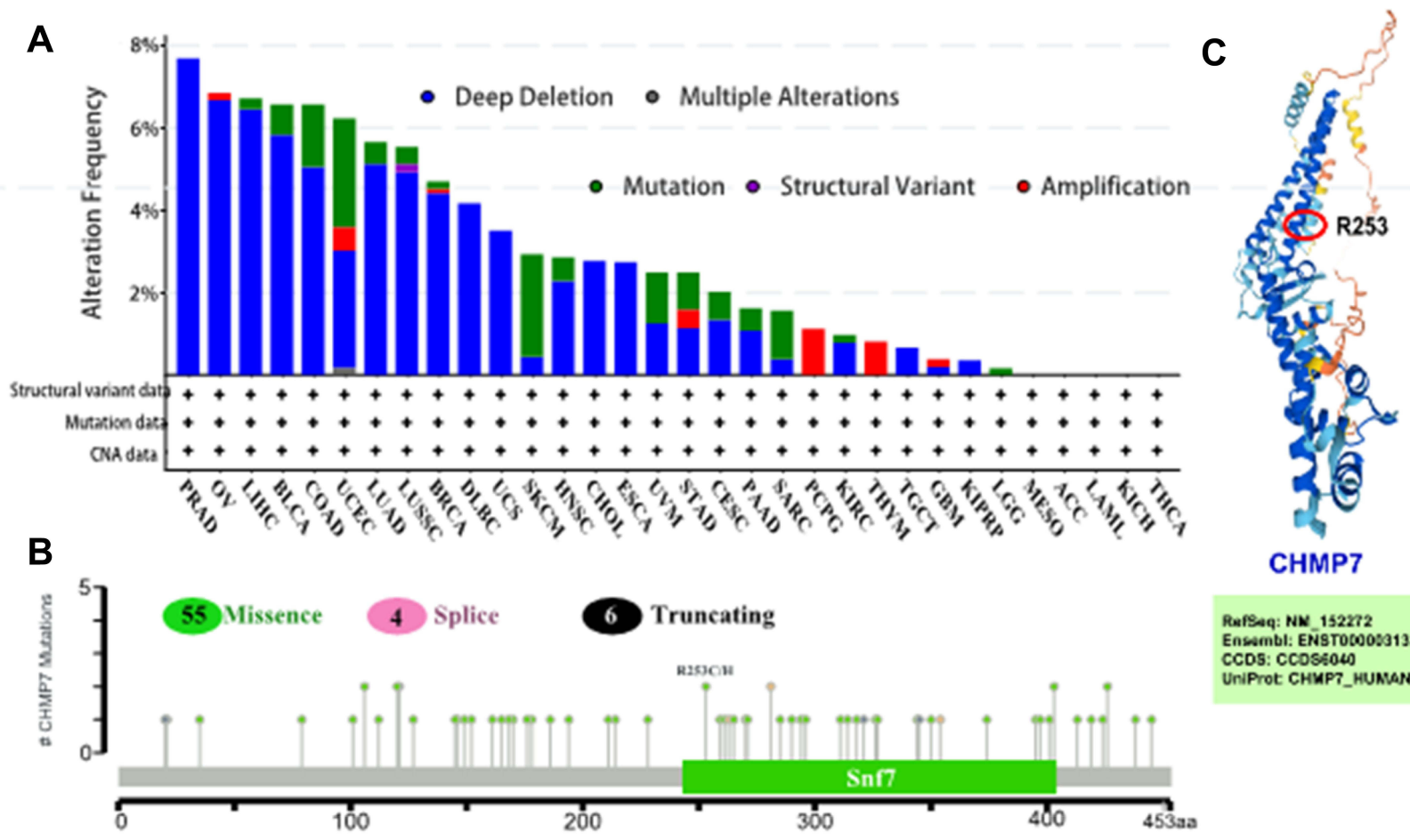

D

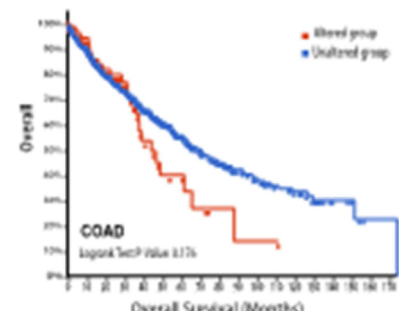

Overal Surveal ONonths

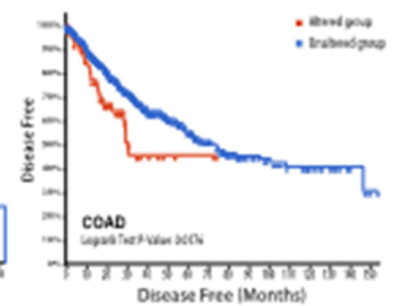

Disease Free |Monthal
$\mathbf{E}$
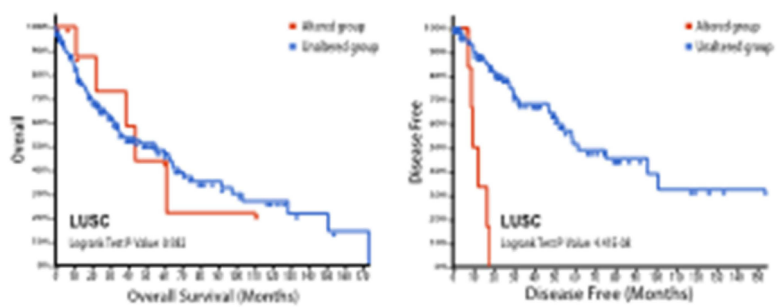

Figure 5 Genetic variation analysis. (A) The main types and frequency of CHMP7 mutation occurrence in each tumor tissue; (B) The main types and loci of CHMP7 mutations; (C) Three-dimensional model of CHMP7 protein; (D) Prognosis of CHMP7 mutations on patients with COAD;(E) Prognosis of CHMP7 mutations on patients with LUSC.

\section{Phosphorylation Analysis}

Protein phosphorylation is one of the most prevalent and important post-translational modifications of proteins in biology, and has been viewed by biologists as a dynamic biological regulatory process since the 1950s. The levels of CHMP7 phosphorylation in BRCA, COAD, OV, KIRC, LUAD and UCEC were analyzed with the CPTAC dataset, and the phosphorylation sites were summarized as in Figure 7A. The most common phosphorylation sites were S307 and S417, both located in the Snf7 region (Figure 7B-H). The results revealed that CHMP7 phosphorylation levels were higher in BRCA ( $\mathrm{P}=2.30 \mathrm{e}-13)$ and KIRC $(\mathrm{P}=2.76 \mathrm{e}-19)$ than in normal tissues, and lower in COAD ( $\mathrm{P}=1.53 \mathrm{e}-09)$, OV $(\mathrm{P}=1.36 \mathrm{e}-03)$, LUAD $(\mathrm{P}=$ 4.15e-13) and UCEC $(\mathrm{P}=2.94 \mathrm{e}-20)$. Snf7 acts a component of the ESCRT-III complex required for the sorting and concentration of proteins resulting in the entry of these proteins into the invaginating vesicles of the MVB.

\section{Immuno-Infiltration Analysis}

The tumor microenvironment (TME) is a complex integrated system formed by the interaction of tumor cells with surrounding tissues and immune cells. The existence of TME can enhance tumor cell proliferation, migration and immune escape ability, thereby promoting the occurrence and development of tumors.

The correlation of different tumor types with each immune checkpoint molecule and mismatch repair gene in the TCGA dataset was analyzed. The results showed 

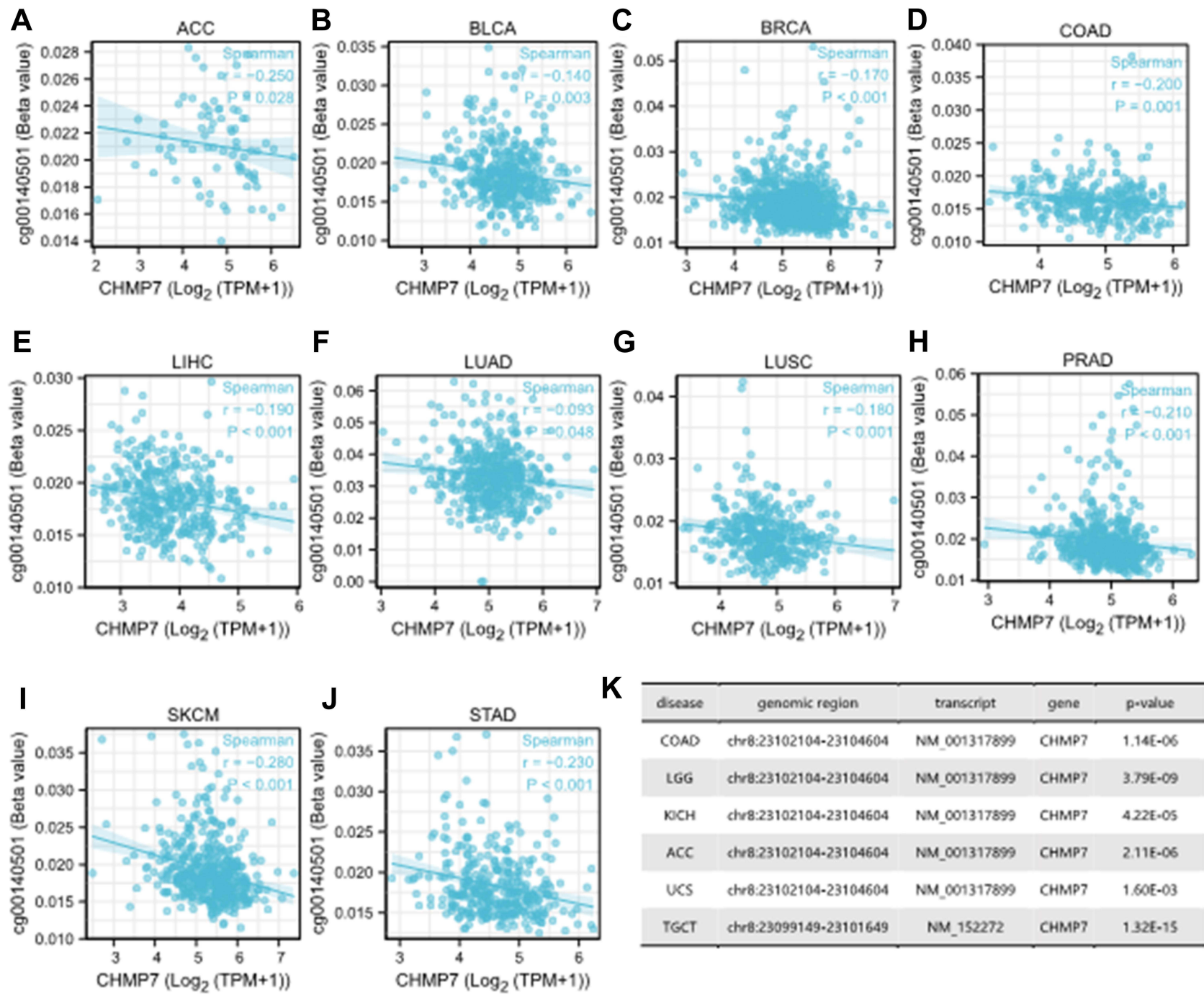

\begin{tabular}{|c|c|c|c|c|}
\hline dsease & genomic region & transcript & gene & p.value \\
\hline COAD & chr8:23102104-23104604 & NM_001317899 & CHMP? & $1.14 E-06$ \\
\hline LGG & chr8:23102104-23104604 & NM_001317899 & CHMP7 & $3.79 \mathrm{E}-\infty 9$ \\
\hline $\mathrm{NCH}$ & chr8:23102104.23104604 & NM_001317899 & CHMP7 & $422 \mathrm{E} \cdot 05$ \\
\hline ACC & chr8:23102104.23104604 & NM_C01317899 & CHMP7 & $2.11 E-06$ \\
\hline USS & chr8:23102104-23104604 & $N_{2} 001317899$ & CHMP7 & $1.60 E-03$ \\
\hline TECT & chrb:23099149-23101649 & NM 152272 & CHMP7 & $1326-15$ \\
\hline
\end{tabular}

Figure 6 Methylation analysis. (A-J) CHMP7 DNA methylation was significantly negatively correlated with probe cg00I4050I in $A C C(R=-0.250 ; P=0.028), B L C A(R=$ $-0.140 ; P=0.003)$, BRCA $(R=-0.170 ; P<0.001), \operatorname{COAD}(R=-0.200 ; P=0.001), \operatorname{LIHC}(R=-0.190 ; P<0.001), L U A D(R=-0.093 ; P=0.048), L U S C(R=-0.180 ; P<$ $0.00 \mathrm{I})$, PRAD $(R=-0.180$; $P<0.00 \mathrm{I})$, SKCM $(R=-0.280$; $\mathrm{P}<0.00 \mathrm{I})$ and STAD $(R=-0.230$; $\mathrm{P}<0.00 \mathrm{I})$; $(\mathrm{K})$ The DiseaseMeth database was used to explore the relationship between CHMP7 DNA methylation and different tumor tissues.

that most of the tumors were significantly correlated with immune checkpoint molecules and mismatch repair genes (Figure 8). Some of the correlation information is summarized in Table 1.

\section{Enrichment Analysis}

To further investigate the molecular mechanism of CHMP7 gene in tumorigenesis, we tried to screen the proteins interacting with CHMP7 and CHMP7 expression-related genes for functional enrichment analysis. The protein-protein interaction (PPI) network of Figure 9A presents the experimentally confirmed proteins included in the String database that interact with CHMP7. 100 genes associated with CHMP7 were obtained from the GEPIA website. GO and KEGG enrichment analysis were performed on the 150 genes (Table 2). They were mainly enriched in viral budding, multivesicular body assembly and multivesicular body organization (BP) while genes in molecular function (MF) was mainly enriched in GTP binding, GTPase activity and structural constituent of cytoskeleton. Genes in cellular component (CC) were mainly enriched in ESCRT I complex, late endosome membrane and ESCRT complex (Figure 9B). A venn diagram was performed to represent the CHMP7 co-expressed genes shared in GEPIA and String and correlation analysis was performed $(\mathrm{R}=0.500$; $\mathrm{P}<0.001$; Figure 9C). KEGG pathway enrichment analysis showed that the main enrichment pathway that make up this modular gene was endocytosis, gap junction and phagosome (Figure 9D; Table 3). 

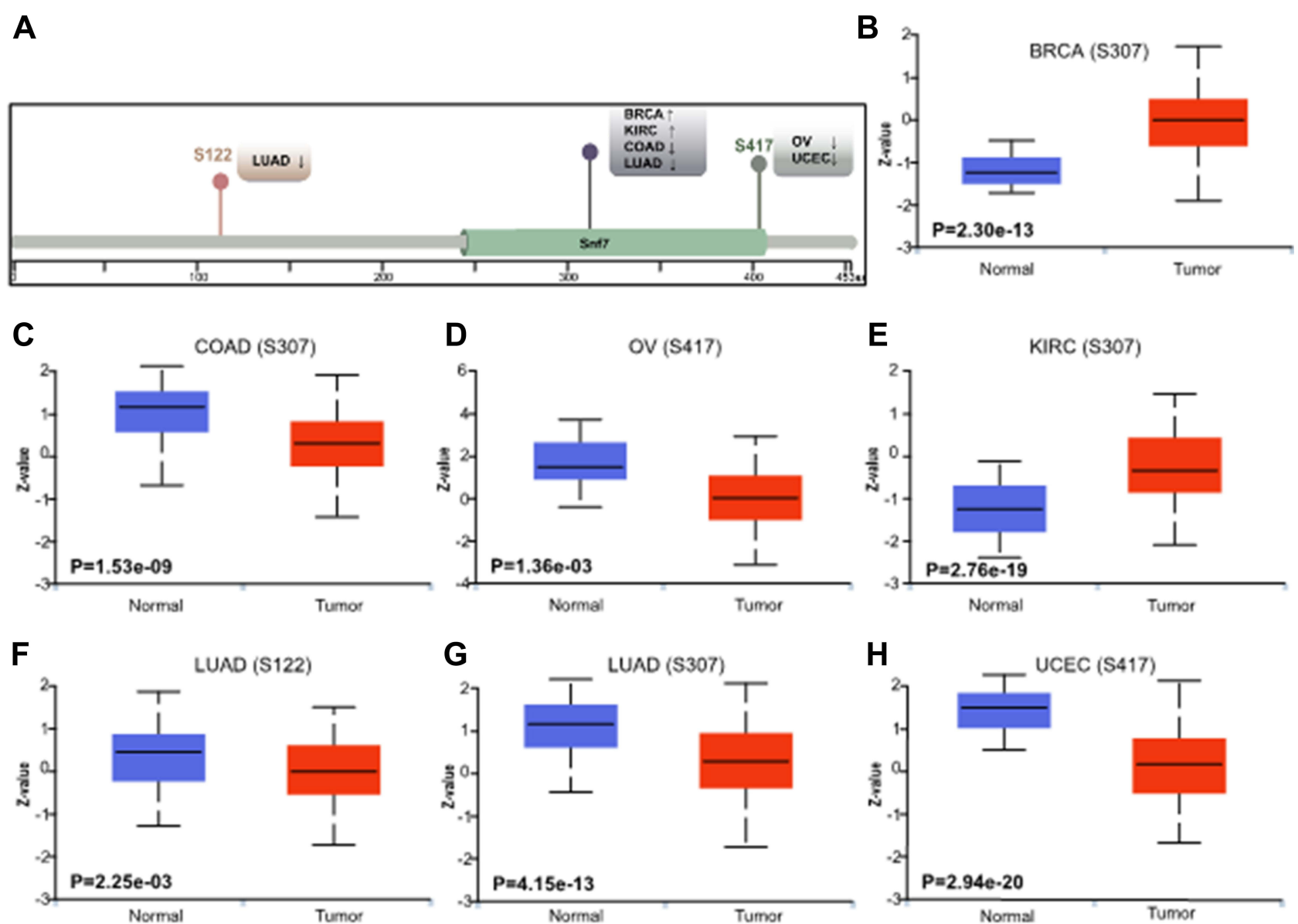

Figure 7 Phosphorylation analysis. (A) Common sites of CHMP7 protein phosphorylation; (B-H) Differential expression of CHMP7 protein in tumor tissues and corresponding normal tissues. IN BRCA and KIRC, tumor tissues were high in phosphorylated proteins; the opposite was true for COAD, LUAD, OV and UCEC.

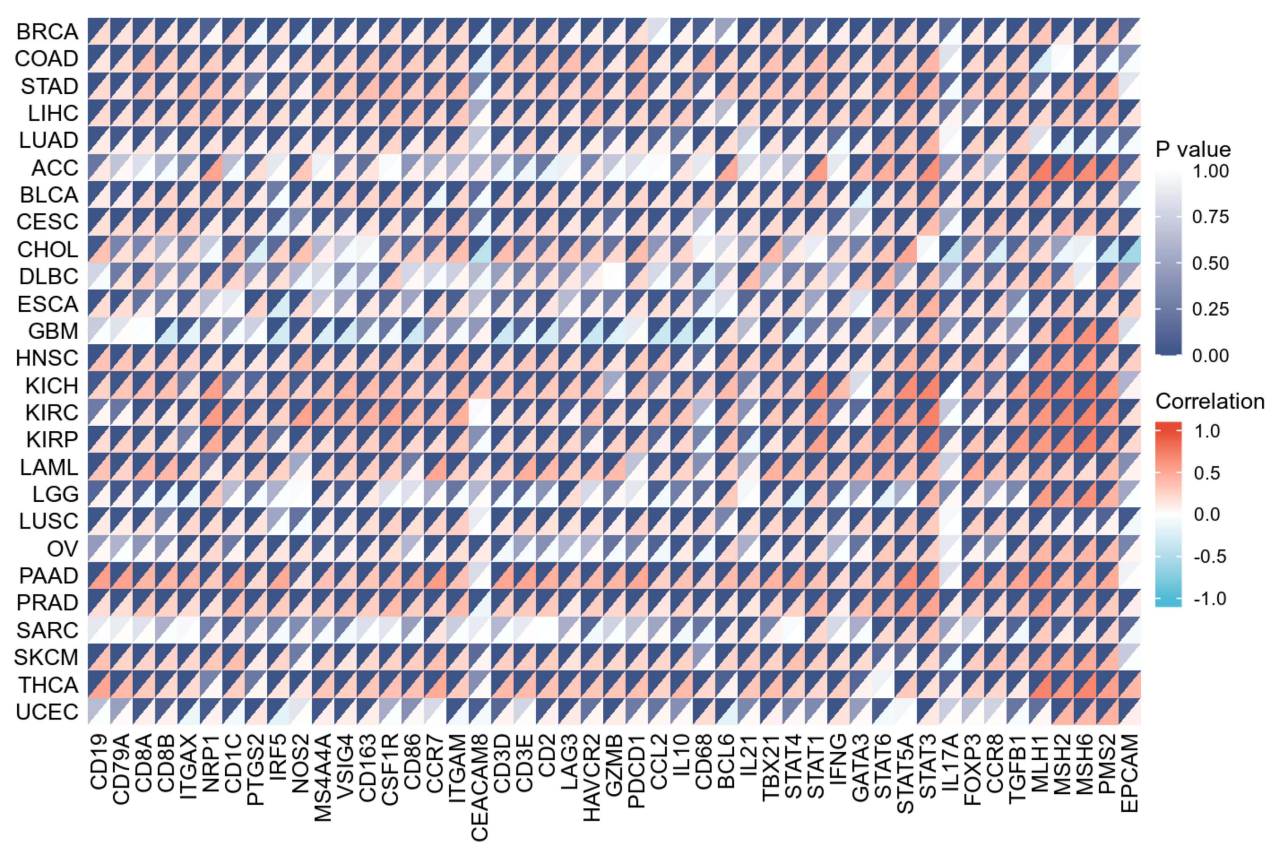

Figure 8 Heat map of CHMP7 correlation analysis with immune checkpoint molecules and mismatch repair genes in multiple tumor tissues. Darker colors represent more significant differences, red represents positive correlation and blue represents negative correlation. 
Table I The Relationship Between CHMP7 and Different Cell Markers of Immune Cells in COAD and LAML

\begin{tabular}{|c|c|c|c|c|c|}
\hline \multirow[t]{2}{*}{ Cell Type } & \multirow[t]{2}{*}{ Gene Marker } & \multicolumn{2}{|c|}{ COAD } & \multicolumn{2}{|c|}{ LAML } \\
\hline & & Cor & $\mathbf{P}$ & Cor & $\mathbf{P}$ \\
\hline \multirow[t]{2}{*}{ B cell } & CDI9 & 0.150 & $<0.001$ & 0.294 & $<0.001$ \\
\hline & CD79A & 0.192 & $<0.001$ & 0.247 & 0.002 \\
\hline \multirow[t]{2}{*}{$\mathrm{CD}^{+} \mathrm{T}$ cell } & CD8A & 0.313 & $<0.001$ & 0.414 & $<0.001$ \\
\hline & $\mathrm{CD} 8 \mathrm{~B}$ & 0.189 & $<0.001$ & 0.410 & $<0.001$ \\
\hline \multirow[t]{3}{*}{ Dentritic celll } & ITGAX & 0.162 & $<0.001$ & 0.143 & 0.080 \\
\hline & NRPI & 0.180 & $<0.001$ & 0.071 & 0.384 \\
\hline & CDIC & 0.107 & 0.019 & 0.158 & 0.053 \\
\hline \multirow[t]{3}{*}{ MI Macrophage } & PTGS2 & 0.097 & 0.034 & 0.163 & 0.045 \\
\hline & IRF5 & 0.031 & 0.496 & 0.166 & 0.041 \\
\hline & NOS2 & 0.164 & $<0.001$ & -0.004 & 0.959 \\
\hline \multirow[t]{3}{*}{ M2 Macrophage } & MS4A4A & 0.134 & 0.003 & 0.180 & 0.027 \\
\hline & VSIG4 & 0.148 & 0.001 & 0.159 & 0.051 \\
\hline & CDI63 & 0.214 & $<0.001$ & 0.197 & 0.015 \\
\hline \multirow[t]{2}{*}{ Monocyte } & CSFIR & 0.181 & $<0.001$ & 0.151 & 0.064 \\
\hline & CD86 & 0.208 & $<0.001$ & 0.045 & 0.584 \\
\hline \multirow[t]{3}{*}{ Neutrophils } & CCR7 & 0.206 & $<0.001$ & 0.495 & $<0.001$ \\
\hline & ITGAM & 0.148 & 0.001 & 0.140 & 0.086 \\
\hline & CEACAM8 & 0.138 & 0.002 & 0.102 & 0.213 \\
\hline \multirow[t]{3}{*}{$\mathrm{T}$ cell } & CD3D & 0.244 & $<0.001$ & 0.238 & 0.003 \\
\hline & CD3E & 0.260 & $<0.001$ & 0.493 & $<0.001$ \\
\hline & $\mathrm{CD} 2$ & 0.282 & $<0.001$ & 0.386 & $<0.001$ \\
\hline \multirow[t]{4}{*}{ T cell exhaustion } & LAG3 & 0.321 & $<0.001$ & 0.224 & 0.006 \\
\hline & HAVCR2 & 0.188 & $<0.001$ & 0.192 & 0.018 \\
\hline & GZMB & 0.059 & 0.196 & 0.374 & $<0.001$ \\
\hline & PDCDI & 0.319 & $<0.001$ & -0.051 & 0.531 \\
\hline \multirow[t]{3}{*}{ TAM } & CCL2 & 0.036 & 0.436 & 0.222 & 0.006 \\
\hline & ILIO & 0.079 & 0.082 & 0.207 & 0.011 \\
\hline & CD68 & 0.303 & $<0.001$ & -0.031 & 0.710 \\
\hline \multirow[t]{2}{*}{ Tfh } & BCL6 & 0.113 & 0.013 & 0.151 & 0.065 \\
\hline & IL2I & 0.159 & $<0.001$ & 0.022 & 0.793 \\
\hline \multirow[t]{4}{*}{ Thl } & TBX2I & 0.304 & $<0.001$ & 0.421 & $<0.001$ \\
\hline & STAT4 & 0.198 & $<0.001$ & 0.277 & $<0.001$ \\
\hline & STATI & 0.277 & $<0.001$ & 0.140 & 0.087 \\
\hline & IFNG & 0.223 & $<0.001$ & 0.277 & $<0.001$ \\
\hline \multirow[t]{3}{*}{ Th2 } & GATA3 & 0.087 & 0.057 & 0.378 & $<0.001$ \\
\hline & STAT6 & 0.097 & 0.033 & 0.140 & 0.086 \\
\hline & STAT5A & 0.129 & 0.005 & 0.070 & 0.390 \\
\hline \multirow[t]{2}{*}{ ThI7 } & STAT3 & $0.24 I$ & $<0.001$ & 0.070 & 0.394 \\
\hline & ILI7A & 0.011 & 0.814 & 0.023 & 0.782 \\
\hline
\end{tabular}

(Continued) 
Table I (Continued).

\begin{tabular}{|l|c|c|c|c|c|}
\hline \multirow{2}{*}{ Cell Type } & \multirow{2}{*}{ Gene Marker } & \multicolumn{2}{|c|}{ COAD } & \multicolumn{2}{c|}{ LAML } \\
\cline { 2 - 5 } & & Cor & P & Cor & P \\
\hline \multirow{2}{*}{ Teg } & FOXP3 & 0.140 & 0.002 & 0.384 & $<0.001$ \\
& CCR8 & 0.166 & $<0.001$ & 0.247 & 0.002 \\
& TGFBI & 0.144 & 0.002 & 0.041 & 0.614 \\
\hline \multirow{3}{*}{ MMR related genes } & MLHI & 0.218 & $<0.001$ & 0.121 & 0.138 \\
& MSH2 & 0.001 & 0.980 & 0.036 & 0.659 \\
& MSH6 & 0.143 & 0.002 & -0.068 & 0.407 \\
& PMS2 & 0.064 & 0.164 & 0.166 & 0.042 \\
& EPCAM & 0.040 & 0.381 & 0.044 & 0.592 \\
\hline
\end{tabular}

\section{Discussion}

CHMP7 plays a significant role in the endosomal sorting pathway. ${ }^{1-3}$ The ESCRT system is the molecular machinery that completes the invagination of endosomal membranes to form MVBs in eukaryotic cells, and its main function is to promote the degradation of membrane proteins tagged with biquitin. ${ }^{4-6}$ CHMP7 can form a complex with ESCRTIII to jointly complete the process of contraction, shear bud neck and final membrane shedding. ${ }^{2}$ The
ESCRT system consists of five core complexes: ESCRT-0, ESCRT-I, ESCRT-II, ESCRT-III and Vps4-Vta1. ${ }^{3,33}$ CHMP7 is mainly involved in the formation of the ESCRT III, which shear the neck of the budding body, thereby releasing the vesicles into the lumen of the endosome and completing the germination process.

Regarding tumor research, various studies have been conducted to demonstrate the oncogenic or oncogenic functions of ESCRT members due to their key role in cell
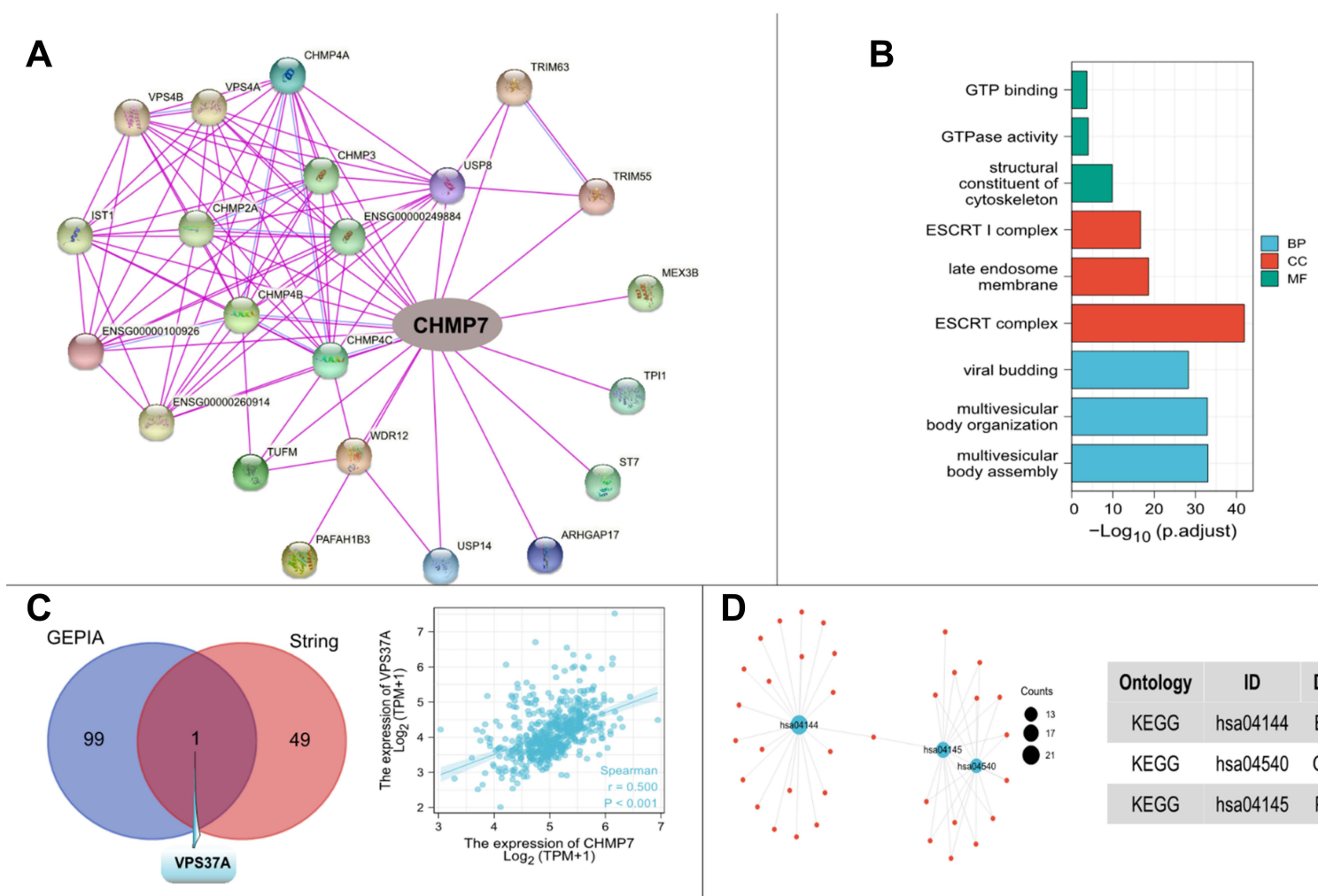

\begin{tabular}{|c|c|c|}
\hline $\begin{array}{c}\text { Ontology } \\
\text { KEGG }\end{array}$ & hsa04144 & Endocytosis \\
\hline KEGG & $h s a 04540$ & Gapjunction \\
\hline KEGG & $h s a 04145$ & Phagosome \\
\hline
\end{tabular}

Figure 9 Functional enrichment analysis. (A) PPI network for CHMP7; (B) GO analysis histogram of CHMP7 and its related genes; (C) Venn diagram of genes associated with CHMP7 from GEPIA and String; (D) Scatter plot of KEGG analysis of CHMP7 and its related genes. 
Table 2 Genes Related to CHMP7 Obtained from GEPIA and String

\begin{tabular}{|l|l|}
\hline Database & Genes \\
\hline GEPIA & VPS37A, KIAA0232, HNRNPDL, ZNF596, CCDC25, \\
& YLPMI, ZNF263, HERCI, CTD-3II3PI6.II, ENTPD4, \\
& STRIPI, ZKSCAN5, COPS4, MLHI, UBTF, INTS9, \\
& PPPIR8, R3HCCI, VPS39, HNRNPM, MORF4LI, \\
& MATR3, BTN2AI, UBR7, NEIL2, USP47, EXOC4, \\
& FAMII4A2, PPP2R2A, TP53BPI, ERCC3, PCMI, \\
& GNPDA2, ABLI, METTLI4, MCPHI, ZFYVEI, \\
& ATP6VIB2, ZBTB49, DHX30, CTD-2530N2I.4, \\
& LDOCIL, ARHGEFI0, SDADIPI, NUDCD3, PPP2CB, \\
& POLR3D, TTI2, ZNF5I0, POLDIP3, ACTRIA, EXTL3, \\
& SART3, ZC3HI0, GEMIN5, CTCF, TATDN2, \\
& SMARCALI, WDR82, MORF4LIPI, VPS4I, ZNFI97, \\
& AGGFI, XPO7, CI Iorf58, DPF2, DCTN6, KBTBD4, \\
& EPC2, EIDI, TAOK2, FAMI68B, LIXIL, C9orfI56, \\
& SARAF, KDM3B, CSTF2T, CCAR2+A2:AI45, \\
& CI2Orf43, HNRNPK, MFAPI, NEK9, FBXWII, \\
& GTF2FI, GPBPI, SAFB, ZBTBI4, TRIM35, TNKS, \\
& RNF20, PPP3CC, RANBP3, FAMI60B2, RADI7, NRFI, \\
& HNRNPUL2, BLOCIS6, RBM23, IP6KI, KCTD20 \\
\hline String & VPS37A, CHMP3, PLIN2, CHMPIB, TUBB6, CHMP6, \\
& TUBB8B, CETNI, CHMP4A, TUBA4A, MAPILC3A, \\
& VPS28, VPS37C, TUBB2A, TSGI0I, TUBAIC, \\
TUBAIA, CHMP2A, TUBB2B, MAPILC3B, UBAPI, \\
MAPILC3C, TUBAL3, STAM, VPS25, VPS37B, VPS36, \\
BABAR4B, TUBBI, CFTR, CHMP7, TUBA3E, TUBA8, \\
HDAC6, MVBI2B, BABARAP, CHMP2B, VPS37D, SNF8 \\
\hline
\end{tabular}

surface receptor degradation. However, few studies have been conducted on CHMP7 in oncology. In this article, we selected TCGA, GEO and CPTAC datasets to explore the expression level of CHMP7 in tumor tissues. The results showed that CHMP7 was lowly expressed in most tumors, and survival analysis by plotting KM curves revealed that the low CHMP7 expression group was associated with poor prognosis in most tumor patients. We also performed CHMP7 genetic mutation analysis, DNA methylation analysis, protein phosphorylation analysis, and immune infiltration analysis to explore the possible mechanisms of CHMP7 low expression in tumor progression.

The analysis of CHMP7 expression in different tumor tissues showed that in most tumor tissues, CHMP7 expression levels were lower than those in normal tissues. Combined with the function of ESCRT-III, which is involved in the formation of CHMP7, it is suggested that the deletion of CHMP7 expression may affect the normal function of ESCRT, leading to impaired inactivation of cytokines or ubiquitinated proteins, further leading to tumorigenesis. Nevertheless, for hematologic malignancies, such as DLBC and LAML, CHMP7 presents high expression. Accordingly, survival analysis of the TCGA and GEO dataset revealed that in most tumor patients, the CHMP7 low-expression group was significantly associated with poor prognosis, while for LAML patients, the CHMP7 high-expression group had a worse prognosis. This result is consistent with the previous analysis of expression differences. Our study also revealed that CHMP7 expression levels were closely correlated with patient tumor stage, and in a variety of solid tumors, patients with lower CHMP7 expression levels showed more advanced tumor stage.

Genetic variation analysis revealed that the most common mutation in CHMP7 is deep deletion, which manifests as missense mutations at the protein level. Most of the mutated sites were located in the Snf7 region, suggesting that Snf7 may be a critical region for CHMP7 to perform its function. Snf7 acts a component of the ESCRT-III complex required for the sorting and concentration of proteins resulting in the entry of these proteins into the invaginating vesicles of the MVB. ${ }^{34} \mathrm{Snf} 7$ is the most abundant ESCRT-III subunit which forms membrane-sculpting filaments with 30 Angstrom periodicity and an exposed cationic membrane-binding surface. ${ }^{35}$ ESCRT-III is critical for late steps in MVB sorting, such as membrane invagination and final cargo sorting and recruitment of late-acting components of the sorting machinery. ${ }^{36}$ Protein phosphorylation analysis verified this conjecture. Protein phosphorylation is the most fundamental, pervasive, and important mechanism that regulates and controls protein viability and function. ${ }^{37}$ Analysis of the CPTAC dataset showed that the majority of the most frequent phosphorylation sites of the CHMP7 protein are also located in the Snf7 region. In summary, Snf7 should be the main region where CHMP7 performs its function, and the deep deletion that occurs in the Snf7 region leads to a frameshift mutation in the CHMP7-encoded protein. The post-transcriptional modification also occurs most frequently in the Snf7 region, and phosphorylation prevents the protein from exercising its function properly, thus leading to tumor progression.

DNA methylation is a form of chemical modification of DNA that can alter genetic expression without altering the DNA sequence. DNA methylation refers to the 
Table 3 Functional Enrichment Analysis of CHMP7 and Its Related Genes

\begin{tabular}{|c|c|c|c|c|c|c|}
\hline Ontology & ID & Description & Gene Ratio & p value & p. Adjust & q value \\
\hline BP & GO:0036258 & Multivesicular body assembly & $20 /|3|$ & $5.20 \mathrm{e}-37$ & $8.70 \mathrm{e}-34$ & $7.68 e-34$ \\
\hline BP & GO:0036257 & Multivesicular body organization & $20 /|3|$ & $1.46 \mathrm{e}-36$ & $1.22 \mathrm{e}-33$ & $1.08 \mathrm{e}-33$ \\
\hline BP & GO:0046755 & Viral budding & $|7 /| 3 \mid$ & $8.51 \mathrm{le}-32$ & $4.75 e-29$ & $4.19 \mathrm{e}-29$ \\
\hline BP & GO:0039702 & Viral budding via host ESCRT complex & $|6 / 13|$ & $2.64 e-31$ & I.I le-28 & $9.75 e-29$ \\
\hline BP & GO:0019068 & Virion assembly & $|7 /| 3 \mid$ & $2.10 \mathrm{e}-27$ & $7.03 e-25$ & $6.20 \mathrm{e}-25$ \\
\hline CC & GO:0036452 & ESCRT complex & $22 / 134$ & $4.87 e-45$ & $1.29 e-42$ & $1.06 \mathrm{e}-42$ \\
\hline $\mathrm{CC}$ & GO:0031902 & Late endosome membrane & $20 / 134$ & $\mid .72 \mathrm{e}-2 \mathrm{|}$ & $2.28 \mathrm{e}-19$ & $1.87 e-19$ \\
\hline $\mathrm{CC}$ & GO:00008I3 & ESCRT I complex & $9 / 134$ & $2.34 \mathrm{e}-19$ & $2.07 e-17$ & $1.70 \mathrm{e}-17$ \\
\hline $\mathrm{CC}$ & GO:0005770 & Late endosome & $22 / 134$ & $3.11 \mathrm{e}-18$ & $2.06 \mathrm{e}-16$ & $1.70 \mathrm{e}-16$ \\
\hline $\mathrm{CC}$ & GO:00008I5 & ESCRT III complex & $8 / 134$ & $1.64 \mathrm{e}-16$ & $7.86 \mathrm{e}-15$ & $6.46 \mathrm{e}-15$ \\
\hline MF & GO:0005200 & Structural constituent of cytoskeleton & $13 / 129$ & $4.79 \mathrm{e}-13$ & $1.42 \mathrm{e}-10$ & $1.30 \mathrm{e}-10$ \\
\hline MF & GO:0003924 & GTPase activity & $13 / 129$ & $7.16 \mathrm{e}-07$ & $1.06 \mathrm{e}-04$ & $9.72 \mathrm{e}-05$ \\
\hline MF & GO:0005525 & GTP binding & $13 / 129$ & $3.51 \mathrm{le}-06$ & $2.03 e-04$ & $1.87 \mathrm{e}-04$ \\
\hline MF & GO:0032550 & Purine ribonucleoside binding & $13 / 129$ & $3.95 \mathrm{e}-06$ & $2.03 e-04$ & $1.87 \mathrm{e}-04$ \\
\hline MF & GO:000I883 & Purine nucleoside binding & $13 / 129$ & $4.30 \mathrm{e}-06$ & $2.03 \mathrm{e}-04$ & $1.87 \mathrm{e}-04$ \\
\hline KEGG & hsa04I44 & Endocytosis & $21 / 76$ & $6.56 \mathrm{e}-15$ & $7.15 \mathrm{e}-13$ & $6.42 \mathrm{e}-13$ \\
\hline KEGG & hsa04540 & Gap junction & $13 / 76$ & $1.07 \mathrm{e}-12$ & $5.84 \mathrm{e}-11$ & $5.25 \mathrm{e}-11$ \\
\hline KEGG & hsa04|45 & Phagosome & $15 / 76$ & $6.96 \mathrm{e}-12$ & $2.38 \mathrm{e}-10$ & $2.13 \mathrm{e}-10$ \\
\hline KEGG & hsa05I32 & Salmonella infection & $18 / 76$ & $8.72 \mathrm{e}-12$ & $2.38 \mathrm{e}-10$ & $2.13 \mathrm{e}-10$ \\
\hline KEGG & hsa050I4 & Amyotrophic lateral sclerosis & $19 / 76$ & $6.03 \mathrm{e}-10$ & I.3le-08 & $1.18 \mathrm{e}-08$ \\
\hline
\end{tabular}

covalent bonding of a methyl group at the cytosine 5 carbon position of a genomic $\mathrm{CpG}$ dinucleotide by the action of DNA methylation transferase. Numerous studies have shown that DNA methylation can cause changes in chromatin structure, DNA conformation, DNA stability and the way DNA interacts with proteins, thereby controlling gene expression. ${ }^{38}$ Analysis by the Diseasemeth database and R software revealed that CHMP7 was negatively associated with methylation probes in a wide range of tumors. Methylation modifications may lead to a decrease in CHMP7 expression or altered protein function, which may be associated with tumorigenesis or progression.

Immune checkpoints are some inhibitory signaling pathways that exist in the immune system. Under normal antitumor immune response, the body maintains a balance of costimulatory and co-inhibitory signals to maintain immune tolerance by regulating the strength of autoimmune response. When the organism is attacked by tumor, it usually blocks the immune checkpoint signaling pathway and thus suppresses autoimmunity, providing opportunities for tumor cells to grow and escape. Tumor immunotherapy is to inhibit and kill tumor cells by mobilizing the body's own immune system, which has become the most promising research direction in the field of tumor therapy. We analyzed the correlation of CHMP7 with immune checkpoint molecules in 33 tumors of TCGA. The heat map demonstrates that CHMP7 expression is significantly associated with immune checkpoint molecules in a variety of tumors, such as CD19, CD79A and IL21 (Figure 6). Lymphocytes that infiltrate into the tumor are called tumor infiltrating lymphocytes (TILs), which include $\mathrm{T}$ lymphocytes, B lymphocytes, macrophages, natural killer (NK) cells, myeloid-derived suppressor cells (MDSC) and dendritic cells (DC) that play a role in antigen presentation. Lymphocytes infiltrating into the tumor mediate the immunosuppressive tumor microenvironment, helping tumor cells to achieve immune escape and promoting tumor malignancy, with tumor infiltrating lymphocytes (TAM) and regulatory T cells (Tregs) playing a major role.

To further investigate the function of CHMP7 and its related genes, GO and KEGG functional aggregation analysis were performed. GO analysis revealed that CHMP7related genes are mainly involved in the composition of the various ESCRT complexes. In terms of molecular function, they mainly bind to GTP, exert GTPase activity and promote MVB assembly. In the KEGG enrichment analysis, the main pathways expressed by CHMP7 and related genes were endocytosis, gap junction and phagosome. The results obtained from the functional enrichment analysis were generally consistent with the above.

In this article, we focused on the correlation between CHMP7 and tumors. And we explored the possible 
mechanisms of CHMP7 action by survival analysis, CHMP7 mutation analysis, DNA methylation analysis, post-translational modification protein phosphorylation analysis, immune infiltration analysis and functional aggregation analysis. The results indicate that deletion of CHMP7 expression, genetic variants, DNA methylation, or post-transcriptional protein modifications resulting in functional alterations are closely associated with tumors.

\section{Conclusion}

Pan-cancer analysis showed that CHMP7 expression was statistically correlated with clinical prognosis, DNA methylation, protein phosphorylation and immune cell infiltration. In conclusion, CHMP7 plays an important role in the development of multiple tumors and may provide new ideas or targets for tumor diagnosis or treatment.

\section{Highlight}

In this article, TCGA, GEO and CPTAC were chosen to analyze the role of CHMP7 in pan-cancer. The role of CHMP7 in pan-cancer was analyzed using $\mathrm{R}$ software and tools such as TIMER, GEPIA, UALCAN, String and DiseaseMeth. This included differential expression analysis, survival analysis, genetic variation analysis, DNA methylation analysis, post-translational modification protein phosphorylation analysis and functional enrichment analysis of CHMP7.

\section{Abbreviation}

CHMP7, Charged multivesicular body protein 7; ESCRT, endosomal sorting complex required for transport; MVBs, multivesicular bodies; ILVs, intralumenal vesicles; EGFR, Epidermal growth factor receptor; SBMA, spinal and bulbar muscular atrophy; TME, tumor microenvironment; HR, hazard ratios; CI, confidence intervals; TILs, tumor infiltrating lymphocytes; TAM, tumor infiltrating lymphocytes; Tregs: regulatory T cells.

\section{Data Sharing Statement}

The datasets used and analyzed during the current study are available from the corresponding author on reasonable request.

\section{Ethics Approval and Consent to Participate}

An application was submitted to the Ethics Review Committee of Jilin University Hospital stating that the data used in this paper were obtained from an online public database and therefore did not involve ethical and moral issues, and a statement of approval was obtained.

\section{Consent for Publication}

Written consent was obtained from the patient for publication of this study and accompanying images.

\section{Funding}

There is no funding to report.

\section{Disclosure}

The authors declare that they have no competing interests.

\section{References}

1. Horii M, Shibata H, Kobayashi R, et al. CHMP7, a novel ESCRT-IIIrelated protein, associates with CHMP4b and functions in the endosomal sorting pathway. Biochem J. 2006;400(1):23-32. PMID:16856878. doi:10.1042/bj20060897

2. Vietri M, Schink KO, Campsteijn C, et al. Spastin and ESCRT-III coordinate mitotic spindle disassembly and nuclear envelope sealing. Nature. 2015;522(7555):231-235. PMID:26040712. doi:10.1038/ nature 14408

3. Hurley JH, Hanson PI. Membrane budding and scission by the ESCRT machinery: it's all in the neck. Nat Rev Mol Cell Biol. 2010;11(8):556-566. PMID:20588296. doi:10.1038/nrm2937

4. Henne WM, Buchkovich NJ, Emr SD. The ESCRT pathway. Dev Cell. 2011;21(1):77-91. PMID:21763610. doi:10.1016/j. devcel.2011.05.015

5. Juan T, Fürthauer M. Biogenesis and function of ESCRT-dependent extracellular vesicles. Semin Cell Dev Biol. 2018;74:66-77. PMID:28807885. doi:10.1016/j.semcdb.2017.08.022

6. Christ L, Raiborg C, Wenzel EM, Campsteijn C, Stenmark H. Cellular functions and molecular mechanisms of the ESCRT membrane-scission machinery. Trends Biochem Sci. 2017;42 (1):42-56. PMID:27669649. doi:10.1016/j.tibs.2016.08.016

7. Bohannon KP, Hanson PI. ESCRT puts its thumb on the nanoscale: fixing tiny holes in endolysosomes. Curr Opin Cell Biol. 2020;65:122-130. PMID:32731154. doi:10.1016/j.ceb.2020.06.002

8. Gatta AT, Carlton JG. The ESCRT-machinery: closing holes and expanding roles. Curr Opin Cell Biol. 2019;59:121-132. PMID:31132588. doi:10.1016/j.ceb.2019.04.005

9. Raiborg C, Stenmark H. The ESCRT machinery in endosomal sorting of ubiquitylated membrane proteins. Nature. 2009;458 (7237):445-452. PMID:19325624. doi:10.1038/nature07961

10. Jimenez AJ, Maiuri P, Lafaurie-Janvore J, Divoux S, Piel M, Perez F. ESCRT machinery is required for plasma membrane repair. Science. 2014;343(6174):1247136. PMID:24482116. doi:10.1126/ science. 1247136

11. Pornillos O, Higginson DS, Stray KM, et al. HIV Gag mimics the Tsg101-recruiting activity of the human Hrs protein. J Cell Biol. 2003;162(3):425-434. PMID:12900394. doi:10.1083/jcb.200302138

12. Lefebvre C, Legouis R, Culetto E. ESCRT and autophagies: endosomal functions and beyond. Semin Cell Dev Biol. 2018;74:21-28. PMID:28807884. doi:10.1016/j.semcdb.2017.08.014

13. Malik B, Devine H, Patani R, La Spada AR, Hanna MG, Greensmith L. Gene expression analysis reveals early dysregulation of disease pathways and links Chmp7 to pathogenesis of spinal and bulbar muscular atrophy. Sci Rep. 2019;9(1):3539. PMID:30837566. doi:10.1038/s41598-019-40118-3 
14. Saksena S, Emr SD. ESCRTs and human disease. Biochem Soc Trans. 2009;37(Pt 1):167-172. PMID:19143624. doi:10.1042/bst0370167

15. Gingras MC, Kazan JM, Pause A. Role of ESCRT component HD-PTP/PTPN23 in cancer. Biochem Soc Trans. 2017;45 (3):845-854. PMID:28620046. doi:10.1042/bst20160332

16. Toyoshima M, Tanaka N, Aoki J, et al. Inhibition of tumor growth and metastasis by depletion of vesicular sorting protein Hrs: its regulatory role on E-cadherin and beta-catenin. Cancer Res. 2007;67(11):5162-5171. PMID:17545595. doi:10.1158/0008-5472. Can-06-2756

17. Oh KB, Stanton MJ, West WW, Todd GL, Wagner KU. Tsg101 is upregulated in a subset of invasive human breast cancers and its targeted overexpression in transgenic mice reveals weak oncogenic properties for mammary cancer initiation. Oncogene. 2007;26 (40):5950-5959. PMID:17369844. doi:10.1038/sj.onc.1210401

18. Liu RT, Huang CC, You HL, et al. Overexpression of tumor susceptibility gene TSG101 in human papillary thyroid carcinomas. Oncogene. 2002;21(31):4830-4837. PMID:12101421. doi:10.1038/ sj.onc. 1205612

19. Nikolova DN, Doganov N, Dimitrov R, et al. Genome-wide gene expression profiles of ovarian carcinoma: identification of molecular targets for the treatment of ovarian carcinoma. Mol Med Rep. 2009;2 (3):365-384. PMID:21475838. doi:10.3892/mmr_00000109

20. Xu Z, Liang L, Wang H, Li T, Zhao M. HCRP1, a novel gene that is downregulated in hepatocellular carcinoma, encodes a growth-inhibitory protein. Biochem Biophys Res Commun. 2003;311 (4):1057-1066. PMID:14623289. doi:10.1016/j.bbrc.2003.10.109

21. Li J, Belogortseva N, Porter D, Park M. Chmp1A functions as a novel tumor suppressor gene in human embryonic kidney and ductal pancreatic tumor cells. Cell Cycle. 2008;7(18):2886-2893. PMID:18787405. doi:10.4161/cc.7.18.6677

22. Wu T, Dai Y. Tumor microenvironment and therapeutic response. Cancer Lett. 2017;387:61-68. PMID:26845449. doi:10.1016/j. canlet.2016.01.043

23. Hinshaw DC, Shevde LA. The tumor microenvironment innately modulates cancer progression. Cancer Res. 2019;79(18):4557-4566. PMID:31350295. doi:10.1158/0008-5472.Can-18-3962

24. Locy H, de Mey S, de Mey W, De Ridder M, Thielemans K, Maenhout SK. Immunomodulation of the tumor microenvironment: turn foe into friend. Front Immunol. 2018;9:2909. PMID:30619273. doi:10.3389/fimmu.2018.02909

25. Blum A, Wang P, Zenklusen JC. SnapShot: TCGA-Analyzed tumors. Cell. 2018;173(2):530. PMID:29625059. doi:10.1016/j. cell.2018.03.059

26. Barrett T, Wilhite SE, Ledoux P, et al. NCBI GEO: archive for functional genomics data sets-update. Nucleic Acids Res. 2013;41 (Database issue):D991-995. PMID:23193258. doi:10.1093/nar/ gks1193
27. Li T, Fan J, Wang B, et al. TIMER: a web server for comprehensive analysis of tumor-infiltrating immune cells. Cancer Res. 2017;77(21): e108-e110. PMID:29092952. doi:10.1158/0008-5472.Can-17-0307

28. Uhlén M, Fagerberg L, Hallström BM, et al. Proteomics. Tissue-based map of the human proteome. Science. 2015;347 (6220):1260419. PMID:25613900. doi:10.1126/science.1260419

29. Choy TK, Wang CY, Phan NN, et al. Identification of Dipeptidyl Peptidase (DPP) family genes in clinical breast cancer patients via an integrated bioinformatics approach. Diagnostics. 2021;11(7). PMID:34359286. doi:10.3390/diagnostics11071204

30. Khoa Ta HD, Tang WC, Phan NN, et al. Analysis of LAGEs family gene signature and prognostic relevance in breast cancer. Diagnostics. 2021;11(4). PMID:33921749. doi:10.3390/ diagnostics 11040726

31. Wu P, Heins ZJ, Muller JT, et al. Integration and analysis of CPTAC proteomics data in the context of cancer genomics in the cBioPortal. Mol Cell Proteomics. 2019;18(9):1893-1898. PMID:31308250. doi:10.1074/mcp.TIR119.001673

32. Ashburner M, Ball CA, Blake JA, et al. Gene ontology: tool for the unification of biology. The gene ontology consortium. Nat Genet. 2000;25(1):25-29. PMID:10802651. doi:10.1038/75556

33. Vietri M, Radulovic M, Stenmark H. The many functions of ESCRTs. Nat Rev Mol Cell Biol. 2020;21(1):25-42. PMID:31705132. doi:10.1038/s41580-019-0177-4

34. Babst M, Katzmann DJ, Estepa-Sabal EJ, Meerloo T, Emr SD. EscrtIII: an endosome-associated heterooligomeric protein complex required for mvb sorting. Dev Cell. 2002;3(2):271-282. PMID:12194857. doi:10.1016/s1534-5807(02)00220-4

35. Tang S, Henne WM, Borbat PP, et al. Structural basis for activation, assembly and membrane binding of ESCRT-III Snf7 filaments. eLife. 2015;4. PMID:26670543. doi:10.7554/eLife.12548

36. Buchkovich NJ, Henne WM, Tang S, Emr SD. Essential N-terminal insertion motif anchors the ESCRT-III filament during MVB vesicle formation. Dev Cell. 2013;27(2):201-214. PMID:24139821. doi:10.1016/j.devcel.2013.09.009

37. Humphrey SJ, James DE, Mann M. Protein phosphorylation: a major switch mechanism for metabolic regulation. Trends Endocrinol Metab. 2015;26(12):676-687. PMID:26498855. doi:10.1016/j. tem.2015.09.013

38. Moore LD, Le T, Fan G. DNA methylation and its basic function. Neuropsychopharmacology. 2013;38(1):23-38. PMID:22781841. doi:10.1038/npp.2012.112
International Journal of General Medicine

\section{Publish your work in this journal}

The International Journal of General Medicine is an international, peer-reviewed open-access journal that focuses on general and internal medicine, pathogenesis, epidemiology, diagnosis, monitoring and treatment protocols. The journal is characterized by the rapid reporting of reviews, original research and clinical studies

\section{Dovepress}

across all disease areas. The manuscript management system is completely online and includes a very quick and fair peer-review system, which is all easy to use. Visit http://www.dovepress.com/ testimonials.php to read real quotes from published authors. 